\title{
LANDAU-LIFSHITZ-BLOCH EQUATION ON RIEMANNIAN MANIFOLD
}

\author{
BOLING GUO, ZONGLIN JIA
}

\begin{abstract}
In this article, we bring in Landau-Lifshitz-Bloch(LLB) equation on $m$ dimensional closed Riemannian manifold and prove that it admits a unique local solution. In addition, if $m \geqslant 3$ and $L^{\infty}$-norm of initial data is sufficiently small, the solution can be extended globally. Moreover, if $m=2$, we can prove that the unique solution is global without assuming small initial data.
\end{abstract}

\section{INTRODUCTION}

Landau-Lifshitz-Gilbert equation describes physical properties of micromagnetic at temperatures below the critical temperature. The equation is as follows:

$$
\frac{\partial m}{\partial t}=\lambda_{1} m \times H_{e f f}-\lambda_{2} m \times\left(m \times H_{e f f}\right)
$$

where $\times$ denotes the vector cross product in $\mathbb{R}^{3}$ and $H_{\text {eff }}$ is effective field while $\lambda_{1}$ and $\lambda_{2}$ are real constants.

However, at high temperature, the model must be replaced by following LandauLifshitz-Bloch equation(LLB)

$$
\frac{\partial u}{\partial t}=\gamma u \times H_{e f f}+L_{1} \frac{1}{|u|^{2}}\left(u \cdot H_{e f f}\right) u-L_{2} \frac{1}{|u|^{2}} u \times\left(u \times H_{e f f}\right)
$$

where $\gamma, L_{1}, L_{2}$ are real numbers and $\gamma>0 . H_{e f f}$ is given by

$$
H_{e f f}=\Delta u-\frac{1}{\chi \|}\left(1+\frac{3 T}{5\left(T-T_{c}\right)}|u|^{2}\right) u .
$$

where $T>T_{c}>0$ and $\chi \|>0$.

Now let us recall some previous results about LLB. In [6], Le consider the case that $L_{1}=L_{2}=: \kappa_{1}>0$. At that time, he rewrites (1.2) as

$$
\frac{\partial u}{\partial t}=\kappa_{1} \Delta u+\gamma u \times \Delta u-\kappa_{2}\left(1+\mu|u|^{2}\right) u
$$

with $\kappa_{2}:=\frac{\kappa_{1}}{\chi \|}$ and $\mu:=\frac{3 T}{5\left(T-T_{c}\right)}$ and assume that $\kappa_{2}, \gamma, \mu$ is positive. Le has proven that above equation with Neumann boundary value conditions has global weak solution(the weak solution here is different from ordinary one). Inspired by Le, in [5] Jia introduces following equation

$$
\left\{\begin{array}{l}
\partial_{t} u=\kappa_{1} \Delta u+\gamma \nabla F(u) \times \Delta u-\kappa_{2}(1+\mu \cdot F(u)) \nabla F(u) \text { in } \Omega \times(0, \infty) \\
\frac{\partial u}{\partial \nu}=0 \text { on } \partial \Omega \times(0, \infty) \\
u(\cdot, 0)=u_{0} \text { in } \Omega
\end{array}\right.
$$


where $\Omega$ is a regular bounded domain of $\mathbb{R}^{d}(d \leqslant 3), \nu$ is outer normal direction of $\partial \Omega$ and $F \in C^{3}\left(\mathbb{R}^{3}\right)$ is a known function. He calls it Generalized Landau-Lifshitz-Bloch equation(GLLB) and gets that (1.4) admits a local strong solution provided $u_{0} \in W^{2,2}\left(\Omega, \mathbb{R}^{3}\right)$ and $\frac{\partial u_{0}}{\partial \nu}=0$. In [4], Guo, Li and Zeng consider the coming LLB equation with initial condition

$$
\left\{\begin{array}{l}
u_{t}=\Delta u+u \times \Delta u-\lambda\left(1+\mu|u|^{2}\right) u \quad \text { in } \mathbb{R}^{d} \times(0, T) \\
u(, 0)=u_{0} \quad \text { in } \mathbb{R}^{d}
\end{array}\right.
$$

where the constant $\lambda, \mu>0$. They prove the existence of smooth solutions of (1.5) in $\mathbb{R}^{2}$ or $\mathbb{R}^{3}$. And a small initial value condition should be added in the latter case.

In this paper, we would like to introduce a equation similar with (1.5) on Riemannian manifold. Before getting to this, we should make some preparation.

Let $\pi:(E, h, D) \longrightarrow(M, g, \nabla)$ denote a smooth vector bundle over an $m$-dimensional smooth closed Riemannian manifold $(M, g, \nabla)$ with $\operatorname{rank}(E)=3$. g means Riemannian metric of $M$ and $\nabla$ is its Levi-Civita connection. $h$ and $D$ are respectively metric and connection of $E$ such that $D h=0$. Sometimes we also write $h$ as $\langle\cdot, \cdot\rangle$.

1.1. $k$-times continuously differentiable section. Suppose $\Gamma(E)$ is the set of all sections in $E$. Under arbitrary local frame $\left\{e_{\alpha}: 1 \leqslant \alpha \leqslant 3\right\}$, a section $s \in \Gamma(E)$ can be written in the form of $s=s^{\alpha} \cdot e_{\alpha}$. If $s^{\alpha}$ is $k$-times continuously differentiable, then we say $s$ is $k$-times continuously differentiable. Since $E$ is smooth, $k$-times continuous differentiability is independent of the choice of local frame. Define

$$
\Gamma^{k}(E):=\{s \in \Gamma(E): s \text { is } k \text {-times continuously differentiable }\} .
$$

1.2. Orientable vector bundle. $E$ is called orientable if there exists an $\omega \in E^{*} \wedge E^{*} \wedge E^{*}$ such that $\omega$ is continuous and for all $p \in M, \omega(p) \neq 0$, where $E^{*}$ is dual bundle of $E$.

Suppose $\left\{e_{1}, e_{2}, e_{3}\right\}$ is a frame of $E$. It is called adapted to the orientation $\omega$ if

$$
\omega\left(e_{1}, e_{2}, e_{3}\right)>0 \text {. }
$$

From now on, we always assume that $E$ is orientable unless otherwise stated.

1.3. Cross product on orientable vector bundle. Suppose $\omega$ is an orientation of $E$. $\left\{e_{\alpha}: 1 \leqslant \alpha \leqslant 3\right\}$ is a local frame of $E$ which is adapted to $\omega$. For any $f_{1}, f_{2} \in \Gamma(E)$, we assume that $f_{1}:=f_{1}^{\alpha} \cdot e_{\alpha}, f_{2}:=f_{2}^{\alpha} \cdot e_{\alpha}$. Their cross product $\times$ is defined as follow

$$
\left(f_{1} \times f_{2}\right)(p):=f_{1}(p) \times f_{2}(p),
$$

where

$$
\begin{aligned}
f_{1}(p) \times f_{2}(p): & =\left(f_{1}^{2}(p) \cdot f_{2}^{3}(p)-f_{2}^{2}(p) \cdot f_{1}^{3}(p)\right) \cdot e_{1}(p) \\
& +\left(f_{2}^{1}(p) \cdot f_{1}^{3}(p)-f_{1}^{1}(p) \cdot f_{2}^{3}(p)\right) \cdot e_{2}(p) \\
& +\left(f_{1}^{1}(p) \cdot f_{2}^{2}(p)-f_{2}^{1}(p) \cdot f_{1}^{2}(p)\right) \cdot e_{3}(p) .
\end{aligned}
$$

It is not hard to verify that $f_{1}(p) \times f_{2}(p)$ does not depend upon the choice of local frames which are adapted to $\omega$. 
1.4. Laplace operator on vector bundle. Define a functional Energy on $\Gamma^{2}(E)$ which is given in the form of

$$
\operatorname{Energy}(X):=\frac{1}{2} \int_{M}|D X|^{2} d M
$$

It is not hard to see that the Euler-Lagrange equation of Energy is

$$
\Delta X:=g^{i j} \cdot\left(D^{2} X\right)\left(\frac{\partial}{\partial x^{i}}, \frac{\partial}{\partial x^{j}}\right)=0,
$$

where $g_{i j}:=g\left(\frac{\partial}{\partial x^{i}}, \frac{\partial}{\partial x^{j}}\right)$ and $\left(g^{i j}\right)$ is the inverse matrix of $\left(g_{i j}\right)$. Then we say that $\Delta$ is the Laplace operator on vector bundle $E$.

1.5. sections depending on time. A section depending on time is a map

$$
V: I \longrightarrow \Gamma(E),
$$

where $I$ is an interval of $\mathbb{R}$. Under arbitrary local frame $\left\{e_{\alpha}: 1 \leqslant \alpha \leqslant 3\right\}, V(t, x)$ can be written as $V(t, x):=V^{\alpha}(t, x) \cdot e_{\alpha}(x)$. If $V^{\alpha}$ is $k$-times continuously differentiable with respect to $t$, we say $V$ is $k$-times continuously differentiable with respect to $t$ and use the symbol $C^{k}(I, \Gamma(E))$ to denote all such $V$. Since $E$ is smooth, differentiability with respect to time is independent of the choice of local frame. Moreover, we define

$$
\left(\partial_{t}^{k} V\right)(t, x):=\left(\partial_{t}^{k} V^{\alpha}\right)(t, x) \cdot e_{\alpha}(x) .
$$

1.6. Sobolev space on vector bundle. Equip $\Gamma^{k}(E)$ with a norm $\|\cdot\|_{H^{k, p}}(p \geqslant 1)$ which is defined as follow

$$
\|s\|_{H^{k, p}}^{p}:=\sum_{i=0}^{k} \int_{M}\left|D^{i} s\right|^{p} d M .
$$

The Sobolev space $H^{k, p}(E)$ is the completion of $\Gamma^{k}(E)$ with respect to the norm $\|\cdot\|_{H^{k, p}}$. For convenience, we also denote $H^{k, 2}$ by $H^{k}$ and $\|\cdot\|_{H^{0, p}}$ by $\|\cdot\|_{p}$.

Having the above preparation, we will give the definition of Landau-Lifshitz-Bloch equation(LLB) on Riemannian manifold.

For any $T>0, \lambda>0$ and $\mu>0$, let us consider a section depending on time $V \in$ $C^{1}\left([0, T], \Gamma^{2}(E)\right)$. LLB is just the following equation

$$
\left\{\begin{array}{l}
\partial_{t} V=\Delta V+V \times \Delta V-\lambda \cdot\left(1+\mu \cdot|V|^{2}\right) V \quad \text { in }(0, T] \times M \\
V(0, \cdot)=V_{0}
\end{array}\right.
$$

Our main results are as follow:

Theorem 1.1. Let $\pi:(E, h, D) \longrightarrow(M, g, \nabla)$ denote a smooth vector bundle over an $m$-dimensional smooth closed Riemannian manifold $(M, g, \nabla)$ with rank $(E)=3$ and $D h=0 . E$ is orientable. Given $l \geqslant m_{0}+1$ (Here $m_{0}:=\left[\frac{m}{2}\right]+3$ and $[q]$ is the integral part of $q$ ) and $V_{0} \in H^{l}(E)$, there is a $T^{*}=T^{*}\left(\left\|V_{0}\right\|_{H^{m_{0}}}\right)>0$ and a unique solution $V$ of (1.6) satisfying that for any $0 \leqslant j \leqslant\left[\frac{l}{\hat{m}}\right]\left(\hat{m}:=\max \left\{2,\left[\frac{m}{2}\right]+1\right\}\right)$ and $\alpha \leqslant l-\hat{m} j$,

$$
\partial_{t}^{j} D^{\alpha} V \in L^{\infty}\left(\left[0, T^{*}\right], L^{2}(E)\right) .
$$


Furthermore, if $V_{0} \in \Gamma^{\infty}(E)$, then $V \in C^{\infty}\left(\left[0, T^{*}\right], \Gamma^{\infty}(E)\right)$.

Theorem 1.2. Let $\pi:(E, h, D) \longrightarrow(M, g, \nabla)$ denote a smooth vector bundle over an $m$-dimensional smooth closed Riemannian manifold $(M, g, \nabla)$ with $\operatorname{rank}(E)=3, m \geqslant 3$ and $D h=0$. E is orientable. For any $T>0$ and $N \geqslant m_{0}+1$, there exists a $\hat{B}_{N}>0$ such that for all $V_{0} \in H^{N}(E)$ with $\left\|V_{0}\right\|_{\infty} \leqslant \hat{B}_{N}$, there is a unique solution of (1.6) satisfying

$$
\partial_{t}^{j} D^{\alpha} V \in L^{\infty}\left([0, T], L^{2}(E)\right) \quad \forall 0 \leqslant j \leqslant\left[\frac{N}{\hat{m}}\right] \forall \alpha \leqslant N-\hat{m} j
$$

and

$$
\partial_{t}^{i} D^{\beta} V \in L^{2}\left([0, T], L^{2}(E)\right) \quad \forall 0 \leqslant i \leqslant\left[\frac{N+1}{\hat{m}+1}\right] \forall \beta \leqslant N+1-(\hat{m}+1) i .
$$

Furthermore, if $V_{0} \in \Gamma^{\infty}(E)$, then $V \in C^{\infty}\left([0, T], \Gamma^{\infty}(E)\right)$.

Theorem 1.3. Let $\pi:(E, h, D) \longrightarrow(M, g, \nabla)$ denote a smooth vector bundle over an 2 -dimensional smooth closed Riemannian manifold $(M, g, \nabla)$ with $\operatorname{rank}(E)=3$ and $D h=0$. E is orientable. For any $T>0, N \geqslant 5$ and $V_{0} \in H^{5}(E)$, there is a unique solution of (1.6) satisfying

$$
\partial_{t}^{j} D^{\alpha} V \in L^{\infty}\left([0, T], L^{2}(E)\right) \quad \forall 0 \leqslant j \leqslant\left[\frac{N}{2}\right] \forall \alpha \leqslant N-2 j
$$

and

$$
\partial_{t}^{i} D^{\beta} V \in L^{2}\left([0, T], L^{2}(E)\right) \quad \forall 0 \leqslant i \leqslant\left[\frac{N+1}{3}\right] \quad \forall \beta \leqslant N+1-3 i .
$$

Furthermore, if $V_{0} \in \Gamma^{\infty}(E)$, then $V \in C^{\infty}\left([0, T], \Gamma^{\infty}(E)\right)$.

\section{Notation And Preliminaries}

In the paper, we appoint that the same indices appearing twice means summing it. And $Q_{1} \lesssim Q_{2}$ implies there is a universal constant $C$ such that $Q_{1} \leqslant C \cdot Q_{2}$.

2.1. Riemannian curvature tensor on vector bundle. Using the connection $D$ on $E$, we can define a tensor $R^{E}$ called Riemannian curvature tensor. For any $X, Y \in T M$ and $s \in \Gamma^{2}(E)$,

$$
R^{E}(X, Y) s:=D_{X} D_{Y} s-D_{Y} D_{X} s-D_{[X, Y]} s .
$$

Let $R^{M}$ be the Riemannian curvature tensor of $M$. Being going to represent $R^{M}$ and $R^{E}$ in local frame, we appoint $\frac{\partial}{\partial x^{i}}$ as $\partial_{i}$. Then,

$$
R^{M}\left(\partial_{i}, \partial_{j}\right) \partial_{r}:=\left(R^{M}\right)_{i j r}^{h} \cdot \partial_{h} \quad \text { and } \quad R^{E}\left(\partial_{i}, \partial_{j}\right) e_{\beta}:=\left(R^{E}\right)_{i j \beta}^{\alpha} \cdot e_{\alpha}
$$

Now we give two tensors

$$
\mathcal{R}^{M}:=\left(\mathcal{R}^{M}\right)_{i j k l} \cdot d x^{i} \otimes d x^{j} \otimes d x^{k} \otimes d x^{l}
$$

and

where

$$
\mathcal{R}^{E}:=\left(\mathcal{R}^{E}\right)_{i j}^{\alpha \beta} \cdot d x^{i} \otimes d x^{j} \otimes e_{\alpha} \otimes e_{\beta}
$$

$$
\left(\mathcal{R}^{M}\right)_{i j k l}:=\left(R^{M}\right)_{i j k}^{h} \cdot g_{h l} \quad \text { and } \quad\left(\mathcal{R}^{E}\right)_{i j}^{\alpha \beta}:=\left(R^{E}\right)_{i j \theta}^{\alpha} \cdot h^{\theta \beta} .
$$

$\left(h_{\alpha \beta}\right)$ is the metric matrix of $h$ and $\left(h^{\theta \beta}\right)$ is its inverse matrix. 
2.2. Cross product of tensors. We also want to introduce cross product between two tensors. Given $S \in \Gamma\left(T^{*} M^{\otimes k} \otimes E\right)$ and $T \in \Gamma\left(T^{*} M^{\otimes l} \otimes E\right)$, let us define

$$
S \times T:=\left(S_{i_{1} \cdots i_{k}} \times T_{j_{1} \cdots j_{l}}\right) \otimes d x^{i_{1}} \otimes \cdots \otimes d x^{i_{k}} \otimes d x^{j_{1}} \otimes \cdots \otimes d x^{j_{l}},
$$

where

$$
S_{i_{1} \cdots i_{k}}:=S\left(\partial_{i_{1}}, \cdots, \partial_{i_{k}}\right) \quad \text { and } \quad T_{j_{1} \cdots j_{l}}:=T\left(\partial_{j_{1}}, \cdots, \partial_{j_{l}}\right) .
$$

It is easy to check

$$
|S \times T| \leqslant|S| \cdot|T|
$$

\subsection{Properties of cross product.}

Theorem 2.1. For any $f_{1}, f_{2} \in \Gamma^{1}(E)$, we have

$$
D\left(f_{1} \times f_{2}\right)=\left(D f_{1}\right) \times f_{2}+f_{1} \times\left(D f_{2}\right) .
$$

Proof. Take any $p \in M$. Then there exists a neighbourhood $U$ and a positive number $\delta$ such that the following map

$$
\exp _{p}: N_{\delta} \triangleq\left\{\hat{v} \in T_{p} M:\|\hat{v}\|<\delta\right\} \longrightarrow U
$$

is a diffeomorphism. Take $v \in T_{p} M$ such that $\|v\|=1$. Define $\gamma_{v}(t):=\exp _{p}(t v)$, where $t \in[0, \delta)$. Now take arbitrary orthonormal basis $\left\{e_{p \alpha}: 1 \leqslant \alpha \leqslant 3\right\}$ in $E_{p}$ which is adapted to $\omega$ and let it move parallelly along $\gamma_{v}$ to get

$$
\left\{e_{\alpha}(t, v): t \in[0, \delta), 1 \leqslant \alpha \leqslant 3\right\} .
$$

Clearly,

$$
w(t):=\omega\left(e_{1}(t, v), e_{2}(t, v), e_{3}(t, v)\right)>0, \quad \forall t \in[0, \delta)
$$

since $w$ is a continuous function with respect to $t$. In the next, let $v$ range all the direction in $T_{p} M$ to obtain

$$
\left\{e_{\alpha}(t, v): t \in[0, \delta), v \in T_{p} M,\|v\|=1,1 \leqslant \alpha \leqslant 3\right\} .
$$

It is a orthonormal frame on $U$ which is adapted to $\omega$ and

$$
\left(D e_{\alpha}\right)(p)=0 \text {. }
$$

Assume that $f_{1}=f_{1}^{\alpha} \cdot e_{\alpha}$ and $f_{2}=f_{2}^{\beta} \cdot e_{\beta}$. Then, (2.3) yields

$$
D f_{1}(p)=d f_{1}^{\alpha}(p) \otimes e_{\alpha}(p) \quad \text { and } \quad D f_{2}(p)=d f_{2}^{\beta}(p) \otimes e_{\beta}(p) .
$$

Recalling the definition of cross product, we have

$$
f_{1} \times f_{2}:=\left(f_{1}^{2} \cdot f_{2}^{3}-f_{2}^{2} \cdot f_{1}^{3}\right) \cdot e_{1}+\left(f_{2}^{1} \cdot f_{1}^{3}-f_{1}^{1} \cdot f_{2}^{3}\right) \cdot e_{2}+\left(f_{1}^{1} \cdot f_{2}^{2}-f_{2}^{1} \cdot f_{1}^{2}\right) \cdot e_{3} .
$$

Therefore, since of (2.3), one can get

$$
\begin{aligned}
{\left[D\left(f_{1} \times f_{2}\right)\right](p): } & =\left[d f_{1}^{2}(p) \cdot f_{2}^{3}(p)+f_{1}^{2}(p) \cdot d f_{2}^{3}(p)-d f_{2}^{2}(p) \cdot f_{1}^{3}(p)-f_{2}^{2}(p) \cdot d f_{1}^{3}(p)\right] \otimes e_{1}(p) \\
(2.4)+\left[d f_{2}^{1}(p) \cdot f_{1}^{3}(p)+f_{2}^{1}(p) \cdot d f_{1}^{3}(p)-d f_{1}^{1}(p) \cdot f_{2}^{3}(p)-f_{1}^{1}(p) \cdot d f_{2}^{3}(p)\right] \otimes e_{2}(p) & \\
& +\left[d f_{1}^{1}(p) \cdot f_{2}^{2}(p)+f_{1}^{1}(p) \cdot d f_{2}^{2}(p)-d f_{2}^{1}(p) \cdot f_{1}^{2}(p)-f_{2}^{1}(p) \cdot d f_{1}^{2}(p)\right] \otimes e_{3}(p) \\
{\left[f_{1} \times\left(D f_{2}\right)\right](p) } & =f_{1}(p) \times\left(D f_{2}\right)(p) \\
& =\left[f_{1}^{2}(p) \cdot d f_{2}^{3}(p)-d f_{2}^{2}(p) \cdot f_{1}^{3}(p)\right] \otimes e_{1}(p) \\
& +\left[d f_{2}^{1}(p) \cdot f_{1}^{3}(p)-f_{1}^{1}(p) \cdot d f_{2}^{3}(p)\right] \otimes e_{2}(p)
\end{aligned}
$$




$$
+\left[f_{1}^{1}(p) \cdot d f_{2}^{2}(p)-d f_{2}^{1}(p) \cdot f_{1}^{2}(p)\right] \otimes e_{3}(p)
$$

and

$$
\begin{aligned}
{\left[\left(D f_{1}\right) \times f_{2}\right](p) } & =\left(D f_{1}\right)(p) \times f_{2}(p) \\
& =\left[d f_{1}^{2}(p) \cdot f_{2}^{3}(p)-f_{2}^{2}(p) \cdot d f_{1}^{3}(p)\right] \otimes e_{1}(p) \\
& +\left[f_{2}^{1}(p) \cdot d f_{1}^{3}(p)-d f_{1}^{1}(p) \cdot f_{2}^{3}(p)\right] \otimes e_{2}(p) \\
& +\left[d f_{1}^{1}(p) \cdot f_{2}^{2}(p)-f_{2}^{1}(p) \cdot d f_{1}^{2}(p)\right] \otimes e_{3}(p) .
\end{aligned}
$$

This theorem follows easily from combining (2.4) with (2.5) and (2.6).

Because of (2.2), it is easy to verify that

$$
D(S \times T)=(D S) \times T+S \times(D T),
$$

provided $S \in \Gamma^{1}\left(T^{*} M^{\otimes k} \otimes E\right)$ and $T \in \Gamma^{1}\left(T^{*} M^{\otimes l} \otimes E\right)$.

2.4. Hamilton's notation. Suppose $k, l, p, q \in \mathbb{N}, S \in T^{*} M^{\otimes k} \otimes E^{\otimes p}$ and $T \in T^{*} M^{\otimes l} \otimes$ $E^{\otimes q}$, where

$$
E^{\otimes p}:=\underbrace{E \otimes \cdots \otimes E}_{p-\text { times }} .
$$

we will write $S * T$, following Hamilton [2], to denote a tensor formed by contraction on some indices of $S \otimes T$ using the coefficients $g^{i j}$ or $h_{\alpha \beta}$.

\section{Theorem 2.2.}

$$
|S * T| \leqslant|S| \cdot|T|
$$

Proof. We will get the above formula in an orthonormal basis of $M$ and an orthonormal basis of $E$.

$$
\begin{aligned}
& |S * T|^{2}=\sum_{\substack{\text { free } \\
\text { indices }}}\left(\sum_{\substack{\text { contracted } \\
\text { indices }}} S_{i_{1} \cdots i_{k}}^{\alpha_{1} \cdots \alpha_{p}} \cdot T_{j_{1} \cdots j_{l}}^{\beta_{1} \cdots \beta_{q}}\right)^{2} \\
& \leqslant \sum_{\substack{\text { free } \\
\text { indices }}}\left[\sum_{\substack{\text { contracted } \\
\text { indices }}}\left(S_{i_{1} \cdots i_{k}}^{\alpha_{1} \cdots \alpha_{p}}\right)^{2}\right] \cdot\left[\sum_{\substack{\text { contracted } \\
\text { indices }}}\left(T_{j_{1} \cdots j_{l}}^{\beta_{1} \cdots \beta_{q}}\right)^{2}\right] \\
& \leqslant\left[\sum_{\substack{\text { free } \\
\text { indices }}} \sum_{\substack{\text { contracted } \\
\text { indices }}}\left(S_{i_{1} \cdots i_{k}}^{\alpha_{1} \cdots \alpha_{p}}\right)^{2}\right] \cdot\left[\sum_{\substack{\text { free } \\
\text { indices contracted }}} \sum_{\text {indices }}\left(T_{j_{1} \cdots j_{l}}^{\beta_{1} \cdots \beta_{q}}\right)^{2}\right] \\
& =|S|^{2} \cdot|T|^{2}
\end{aligned}
$$

Because we do not specifically illustrate which indices are contracted, we have to appoint that

$$
S_{1} * T_{1}-S_{2} * T_{2}:=S_{1} * T_{1}+S_{2} * T_{2}
$$


We will use the symbol $\mathfrak{q}_{s}\left(T_{1}, \cdots, T_{r}\right)$ for a polynomial in the tensors $T_{1}, \cdots, T_{r}$ and their iterated covariant derivatives with the $*$ product like

$$
\mathfrak{q}_{s}\left(T_{1}, \cdots, T_{r}\right):=\sum_{j_{1}+\cdots+j_{r}=s} c_{j_{1} \cdots j_{r}} \cdot D^{j_{1}} T_{1} * \cdots * D^{j_{r}} T_{r}
$$

where for $1 \leqslant i \leqslant r, T_{i} \in \Gamma^{j_{i}}\left(T^{*} M^{\otimes t_{i}} \otimes E^{\otimes q_{i}}\right)$ and $c_{j_{1} \cdots j_{r}}$ are some universal constants.

2.5. Ricci identity. Given $s \in \Gamma^{2}\left(T^{*} M^{\otimes k} \otimes E\right)$, it is obvious to see that $s$ can be written as follow

$$
s:=s_{i_{1} \cdots i_{k}}^{\alpha} \cdot d x^{i_{1}} \otimes \cdots \otimes d x^{i_{k}} \otimes e_{\alpha} .
$$

We denote $D s$ in the form of components

$$
D s:=s_{i_{1} \cdots i_{k}, p}^{\alpha} \cdot d x^{i_{1}} \otimes \cdots \otimes d x^{i_{k}} \otimes d x^{p} \otimes e_{\alpha} .
$$

At some time, we also employ the coming convention

$$
D s:=s_{i_{1} \cdots i_{k}, p} \cdot d x^{i_{1}} \otimes \cdots \otimes d x^{i_{k}} \otimes d x^{p} .
$$

Thanks to the above agreement, Ricci identity is conveniently represented in the next theorem.

\section{Theorem 2.3.}

$$
\begin{aligned}
& s_{i_{1} \cdots i_{k}, p q}^{\alpha}-s_{i_{1} \cdots i_{k}, q p}^{\alpha} \\
= & \sum_{l=1}^{k} s_{i_{1} \cdots i_{l-1} h i_{l+1} \cdots i_{k}}^{\alpha} \cdot\left(R^{M}\right)_{p q i_{l}}^{h}-s_{i_{1} \cdots i_{k}}^{\beta} \cdot\left(R^{E}\right)_{p q \beta}^{\alpha} \\
= & k \cdot s * \mathcal{R}^{M}+s * \mathcal{R}^{E} .
\end{aligned}
$$

Proof. The proof is straightforward if one takes normal coordinates. So we omit it.

Given $V \in \Gamma^{k+1}(E)$ and $S \in \Gamma^{k+1}\left(T^{*} M \otimes E\right)$, by Theorem 2.3 and induction, the following formulas are easy.

Formula 1.

There exist $a_{i j} \in \mathbb{Z}$ and $b_{r l} \in \mathbb{Z}$ such that

$$
\begin{aligned}
V_{, p i_{1} \cdots i_{k}}-V_{, i_{1} \cdots i_{k} p} & =\sum_{i+j=k-1} a_{i j} \cdot D^{i} V * D^{j} \mathcal{R}^{E}+\sum_{r+l=k-2} b_{r l} \cdot D^{r+1} V * \nabla^{l} \mathcal{R}^{M} \\
& =\mathfrak{q}_{k-1}\left(V, \mathcal{R}^{E}\right)+\mathfrak{q}_{k-2}\left(D V, \mathcal{R}^{M}\right)
\end{aligned}
$$

\section{Formula 2.}

There exist $a_{i j} \in \mathbb{Z}$ and $b_{r l} \in \mathbb{Z}$ such that

$$
\begin{aligned}
S_{p, q i_{1} \cdots i_{k}}-S_{p, i_{1} \cdots i_{k} q} & =\sum_{i+j=k-1} a_{i j} \cdot D^{i} S * D^{j} \mathcal{R}^{E}+\sum_{r+l=k-1} b_{r l} \cdot D^{r} S * \nabla^{l} \mathcal{R}^{M} \\
& =\mathfrak{q}_{k-1}\left(S, \mathcal{R}^{E}\right)+\mathfrak{q}_{k-1}\left(S, \mathcal{R}^{M}\right)
\end{aligned}
$$


2.6. Interpolation for sections. We shall prove Gagliardo-Nirenberg inequality of sections on vector bundle.

Theorem 2.4. $(M, g)$ is a $m$-dimensional smooth closed Riemannian manifold. $(E, h, D)$ is a smooth vector bundle over $M$ with $D h=0 . \operatorname{rank}(E)$ may not be 3 and $E$ may not be orientable. Let $T$ be a smooth section of $E$. Given $s \in \mathbb{R}^{+}$and $j \in \mathbb{Z}^{+}$, we will have

$$
\left\|D^{j} T\right\|_{\frac{2 s}{l}} \leqslant C(m, s, k, j) \cdot\left\|D^{k} T\right\|_{\frac{2 s}{l+k-j}}^{\frac{j}{k}} \cdot\|T\|_{\frac{2 s}{l-j}}^{1-\frac{j}{k}},
$$

provided $k \in[j, \infty) \cap \mathbb{Z}, l \in[1, s] \cap[j, s+j+1-k] \cap \mathbb{Z}$.

Proof. Apply induction for $j$.

Step 1: When $j=1$, (2.11) is equivalent to

$$
\|D T\|_{\frac{2 s}{l}} \leqslant C(m, s, k) \cdot\left\|D^{k} T\right\|_{\frac{2 s}{l+k-1}}^{\frac{1}{k}} \cdot\|T\|_{\frac{2 s}{l-1}}^{1-\frac{1}{k}},
$$

for all $l \in[1, s] \cap[1, s+2-k] \cap \mathbb{Z}$. In order to show (2.12), we use induction for $k$.

When $k=1$, (2.12) holds obviously.

When $k=2$, by 12.1 Theorem of [2] we know (2.12) holds.

Assume that for $2 \leqslant \hat{k} \leqslant k$, we obtain

$$
\|D T\|_{\frac{2 s}{l}} \leqslant C_{1}(m, s, \hat{k}) \cdot\left\|\left.D^{\hat{k}} T\right|_{\frac{2 s}{l+\hat{k}-1}} ^{\frac{1}{\hat{k}}} \cdot\right\| T \|_{\frac{2 s}{l-1}}^{1-\frac{1}{k}},
$$

provided $l \in[1, s] \cap[1, s+2-\hat{k}] \cap \mathbb{Z}$.

When $\hat{k}=k+1$, pick any $l \in[1, s] \cap[1, s+2-(k+1)] \cap \mathbb{Z}$. Clearly,

$$
l+1 \in[1, s] \cap[1, s+2-k] \cap \mathbb{Z},
$$

since $k \geqslant 2$. Using induction hypothesis, we get

$$
\left\|D^{2} T\right\|_{\frac{2 s}{l+1}} \leqslant C_{2}(m, s, k) \cdot\left\|\left.D^{k}(D T)\right|_{\frac{2 s}{l+k}} ^{\frac{1}{k}} \cdot\right\| D T \|_{\frac{2 s}{l}}^{1-\frac{1}{k}} .
$$

Because $1 \leqslant l \leqslant s+2-(k+1)<s$, using induction hypothesis for $k=2$ gives

$$
\|D T\|_{\frac{2 s}{l}} \leqslant C(m, s) \cdot\left\|D^{2} T\right\|_{\frac{2 s}{l+1}}^{\frac{1}{2}} \cdot\|T\|_{\frac{2 s}{l-1}}^{\frac{1}{2}} \cdot
$$

Combing (2.13) with (2.14) yields

$$
\|D T\|_{\frac{2 s}{l}} \leqslant C_{3}(m, s, k) \cdot\left\|D^{k+1} T\right\|_{\frac{2 s}{l+k}}^{\frac{1}{2 k}} \cdot\|D T\|_{\frac{2 s}{l}}^{\frac{1}{2}\left(1-\frac{1}{k}\right)} \cdot\|T\|_{\frac{2 s}{l-1}}^{\frac{1}{2}},
$$

which implies

$$
\|D T\|_{\frac{2 s}{l}} \leqslant C(m, s, k+1) \cdot\left\|D^{k+1} T\right\|_{\frac{2 s}{l+k}}^{\frac{1}{k+1}} \cdot\|T\|_{\frac{2 s}{l-1}}^{1-\frac{1}{k+1}} .
$$

Step 2: Suppose that for all the indices not greater than $j$, (2.11) is true. Now we consider $j+1$. At this moment, we take any $k \in[j+1, \infty) \cap \mathbb{Z}$ and any $l \in[1, s] \cap[j+$ $1, s+j+2-k] \cap \mathbb{Z}$. It is easy to deduce that

$$
k-1 \in[j, \infty) \cap \mathbb{Z} \quad \text { and } \quad l \in[1, s] \cap[j, s+j+1-(k-1)] \cap \mathbb{Z} .
$$

Using induction hypothesis leads to

$$
\left\|D^{j}(D T)\right\|_{\frac{2 s}{l}} \leqslant C_{1}(m, s, k, j) \cdot\left\|\left.D^{k-1}(D T)\right|_{\frac{2 s}{l+k-1-j}} ^{\frac{j}{k-1}} \cdot\right\| D T \|_{\frac{2 s}{l-j}}^{1-\frac{j}{k-1}} .
$$


Since $l-j \in[1, s] \cap[1, s+2-k] \cap \mathbb{Z}$, by Step 1 we have

$$
\|D T\|_{\frac{2 s}{l-j}} \leqslant C(m, s, k) \cdot\left\|D^{k} T\right\|_{\frac{2 s}{l-j+k-1}}^{\frac{1}{k}} \cdot\|T\|_{\frac{2 s}{l-j-1}}^{1-\frac{1}{k}} .
$$

Combining (2.15) with (2.16) gives

$$
\begin{aligned}
\left\|D^{j+1} T\right\|_{\frac{2 s}{l}} & \leqslant C(m, s, k, j+1) \cdot\left\|D^{k} T\right\|_{\frac{2 s}{l+k-1-j}}^{\frac{j}{k-1}} \cdot\left\|D^{k} T\right\|_{\frac{2 s}{l+k-j-1}}^{\frac{1}{k}\left(1-\frac{j}{k-1}\right)} \cdot\|T\|_{\frac{2 s}{l-j-1}}^{\left(1-\frac{1}{k}\right)\left(1-\frac{j}{k-1}\right)} \\
& =C(m, s, k, j+1) \cdot\left\|D^{k} T\right\|_{\frac{2 s}{l+k-1-j}}^{\frac{j+1}{k}} \cdot\|T\|_{\frac{2 s}{l-j-1}}^{1-\frac{j+1}{k}} .
\end{aligned}
$$

This completes the proof.

Theorem 2.5. $(M, g)$ is a m-dimensional smooth closed Riemannian manifold. $(E, h, D)$ is a smooth vector bundle over $M$ with $D h=0$. rank $(E)$ may not be 3 and $E$ may not be orientable. Let $T$ be a smooth section of $E$. If $r, q \geqslant 2$, then there is a universal constant $C=C(m, r, q, j, k)$ such that

$$
\left\|D^{j} T\right\|_{p} \leqslant C \cdot\left\|D^{k} T\right\|_{r}^{\frac{j}{k}} \cdot\|T\|_{q}^{1-\frac{j}{k}}
$$

provided

$$
1 \leqslant j \leqslant k \quad \text { and } \quad \frac{k}{p}=\frac{j}{r}+\frac{k-j}{q} .
$$

Proof. We consider 3 cases.

Case 1: When $2 \leqslant r<q \leqslant \infty$, there exist $s$ and $l$ such that

$$
q=\frac{2 s}{l-j} \quad \text { and } \quad r=\frac{2 s}{l+k-j} .
$$

Since

$$
\frac{k}{p}=\frac{j}{r}+\frac{k-j}{q},
$$

we have $p=\frac{2 s}{l}$. From Theorem 2.4 it follows that

which means

$$
\left\|D^{j} T\right\|_{\frac{2 s}{l}} \leqslant C(m, s, k, j) \cdot\left\|D^{k} T\right\|_{\frac{2 s}{l+k-j}}^{\frac{j}{k}} \cdot\|T\|_{\frac{2 s}{l-j}}^{1-\frac{j}{k}}
$$

$$
\left\|D^{j} T\right\|_{p} \leqslant C(m, r, q, j, k) \cdot\left\|D^{k} T\right\|_{r}^{\frac{j}{k}} \cdot\|T\|_{q}^{1-\frac{j}{k}}
$$

Case 2: When $2 \leqslant q<r \leqslant \infty$, the proof is similar.

Case 3: When $2 \leqslant q=r$, clearly we have $p=q=r$. From 12.1 Theorem in [2] it follows that

which implies

$$
\|D T\|_{p} \leqslant C(m, p) \cdot\left\|D^{2} T\right\|_{p}^{\frac{1}{2}} \cdot\|T\|_{p}^{\frac{1}{2}}
$$

$$
\left\|D^{j} T\right\|_{p} \leqslant C(m, p) \cdot\left\|D^{j+1} T\right\|_{p}^{\frac{1}{2}} \cdot\left\|D^{j-1} T\right\|_{p}^{\frac{1}{2}} .
$$

Let $f(j):=\left\|D^{j} T\right\|_{p}$. It is easy to check that $f$ meets the condition of 12.5 Corollary in [2]. Then we conclude this theorem. 


\section{Proof of Theorem 1.1}

Given any $T>0$, define an operator

$$
P: C^{1}\left([0, T], \Gamma^{2}(E)\right) \longrightarrow C([0, T], \Gamma(E)),
$$

here

$$
P(V):=\partial_{t} V-\Delta V-V \times \Delta V-\lambda\left(1+\mu|V|^{2}\right) V .
$$

It is not difficult to check that the leading coefficient of the linearised operator of $P$ meets Legendre-Hadamard condition. By Main Theorem 1 in page 3 of [1] we know (1.6) admits a unique local smooth solution $V$ provided $V_{0} \in \Gamma^{\infty}(E)$.

In the sequel, we would like to know the lower bound of maximal existence time $T_{\max }$ of the above smooth solution. Our strategy is to deduce a Gronwall inequality. That is to say, we shall control $\frac{d}{d t}\|V(t)\|_{H^{l}}^{2}$. Before getting to this, it is important to obtain an upper bound of $\|V(t)\|_{\infty}$.

Taking inner product with $|V|^{p-2} V(p>2)$ in (1.6), and integrating the result over $M$, we get

$$
\begin{aligned}
\int_{M}|V|^{p-2}\left\langle V, \partial_{t} V\right\rangle d M & =\int_{M}|V|^{p-2}\langle V, \Delta V\rangle d M-\lambda \int_{M}\left(1+\mu|V|^{2}\right)|V|^{p} d M \\
& \leqslant-\int_{M}|V|^{p-2} \cdot|D V|^{2} d M-(p-2) \int_{M}|V|^{p-4} \cdot|\langle V, D V\rangle|^{2} d M \leqslant 0 .
\end{aligned}
$$

The left hand side of the above inequality is $\frac{1}{p} \frac{d}{d t}\|V(t)\|_{p}^{p}$, so this inequality means

$$
\|V(t)\|_{p} \leqslant\left\|V_{0}\right\|_{p} \quad \forall t \in\left[0, T_{\max }\right) .
$$

Taking the limit $p \rightarrow \infty$ leads to

$$
\|V(t)\|_{\infty} \leqslant\left\|V_{0}\right\|_{\infty} \quad \forall t \in\left[0, T_{\max }\right) .
$$

Given $k \geqslant 1$, recalling our appointment (2.8), we have the next identity

$$
\begin{aligned}
\frac{1}{2} \frac{d}{d t} \int_{M}\left|D^{k} V\right|^{2} d M= & \int_{M} g^{p q} g^{i_{1} j_{1}} \cdots g^{i_{k} j_{k}}\left\langle V_{, j_{1} \cdots j_{k}}, V_{, p q i_{1} \cdots i_{k}}\right\rangle d M \\
& +\int_{M} g^{p q} g^{i_{1} j_{1}} \cdots g^{i_{k} j_{k}}\left\langle V_{, j_{1} \cdots j_{k}},\left(V \times V_{, p}\right)_{, q i_{1} \cdots i_{k}}\right\rangle d M \\
& -\lambda \cdot \mu \int_{M} g^{i_{1} j_{1}} \cdots g^{i_{k} j_{k}}\left\langle V_{, j_{1} \cdots j_{k}},\left(|V|^{2} V\right)_{, i_{1} \cdots i_{k}}\right\rangle d M \\
& -\lambda \int_{M}\left|D^{k} V\right|^{2} d M
\end{aligned}
$$

Applying (2.9) and (2.10) to exchange the order of derivatives yields

$$
\begin{aligned}
\frac{1}{2} \frac{d}{d t} \int_{M}\left|D^{k} V\right|^{2} d M= & -\int_{M}\left|D^{k+1} V\right|^{2} d M+\int_{M} D^{k} V * \mathfrak{q}_{k}\left(V, \mathcal{R}^{E}\right) d M \\
& +\int_{M} D^{k} V * \mathfrak{q}_{k-1}\left(D V, \mathcal{R}^{M}\right) d M \\
& -\int_{M} g^{p q} g^{i_{1} j_{1}} \cdots g^{i_{k} j_{k}}\left\langle V_{, q j_{1} \cdots j_{k}},\left(V \times V_{, p}\right)_{, i_{1} \cdots i_{k}}\right\rangle d M
\end{aligned}
$$




$$
\begin{aligned}
& -\int_{M} D^{k} V * \mathfrak{q}_{k-1}\left(V \times D V, \mathcal{R}^{M}\right) d M \\
& -\int_{M} D^{k} V * \mathfrak{q}_{k-1}\left(V \times D V, \mathcal{R}^{E}\right) d M \\
& -\lambda \int_{M}\left|D^{k} V\right|^{2} d M-\lambda \mu \int_{M}|V|^{2} \cdot\left|D^{k} V\right|^{2} d M \\
& -\lambda \mu \sum_{i+j=k-1} b_{i j} \cdot \int_{M} D^{k} V * D^{i+1}\left(|V|^{2}\right) * D^{j} V d M \\
& +\int_{M} \mathfrak{q}_{k}\left(V, \mathcal{R}^{E}\right) * D^{k-1}(V \times D V) d M \\
& +\int_{M} \mathfrak{q}_{k-1}\left(D V, \mathcal{R}^{M}\right) * D^{k-1}(V \times D V) d M,
\end{aligned}
$$

here $b_{i j} \in \mathbb{Z}^{+}$are some universal constants. Note that

$$
g^{p q} g^{i_{1} j_{1}} \cdots g^{i_{k} j_{k}}\left\langle V_{, q j_{1} \cdots j_{k}},\left(V \times V_{, p}\right)_{, i_{1} \cdots i_{k}}\right\rangle=\sum_{i+j=k-1} a_{i j} \cdot D^{k+1} V *\left(D^{i+1} V \times D^{j+1} V\right)
$$

where $a_{i j} \in \mathbb{Z}^{+}$are some universal constants. Taking norms on the right hand side of (3.2) leads to

$$
\begin{aligned}
\frac{1}{2} \frac{d}{d t} \int_{M}\left|D^{k} V\right|^{2} d M \leqslant & -\int_{M}\left|D^{k+1} V\right|^{2} d M+\sum_{i=0}^{k} C_{i} \int_{M}\left|D^{k} V\right| \cdot\left|D^{i} V\right| d M \\
& +\sum_{i+j=k-1} a_{i j} \int_{M}\left|D^{k+1} V\right| \cdot\left|D^{i+1} V\right| \cdot\left|D^{j+1} V\right| d M \\
& +\sum_{i=0}^{k-1} \bar{C}_{i} \int_{M}\left|D^{k} V\right| \cdot\left|D^{i}(V \times D V)\right| d M \\
& +\lambda \mu \sum_{i+j=k-1} b_{i j} \int_{M}\left|D^{k} V\right| \cdot\left|D^{i+1}\left(|V|^{2}\right)\right| \cdot\left|D^{j} V\right| d M \\
& +\sum_{i=0}^{k} C_{i} \int_{M}\left|D^{i} V\right| \cdot\left|D^{k-1}(V \times D V)\right| d M,
\end{aligned}
$$

where $C_{i}$ and $\bar{C}_{i}$ depend upon $\mathcal{R}^{M}, \mathcal{R}^{E}$ and their covariant differentiations. Applying (2.1) and (2.7) yields

$$
\begin{aligned}
\frac{1}{2} \frac{d}{d t} \int_{M}\left|D^{k} V\right|^{2} d M \leqslant & -\int_{M}\left|D^{k+1} V\right|^{2} d M+\sum_{i+j=k-1} a_{i j} \int_{M}\left|D^{k+1} V\right| \cdot\left|D^{i+1} V\right| \cdot\left|D^{j+1} V\right| d M \\
& +\tilde{C}_{k}\left\{|| D^{k} V\left\|_{2} \cdot|| V\right\|_{H^{k}}+\sum_{0 \leqslant r+q \leqslant k-1} \int_{M}\left|D^{k} V\right| \cdot\left|D^{r} V\right| \cdot\left|D^{q+1} V\right| d M\right. \\
& +\sum_{r+q+j=k} \int_{M}\left|D^{k} V\right| \cdot\left|D^{r} V\right| \cdot\left|D^{q} V\right| \cdot\left|D^{j} V\right| d M
\end{aligned}
$$




$$
\left.+\sum_{i=0}^{k} \sum_{r+q=k-1} \int_{M}\left|D^{i} V\right| \cdot\left|D^{r} V\right| \cdot\left|D^{q+1} V\right| d M\right\}
$$

where $\tilde{C}_{k}$ depends upon $\mathcal{R}^{M}, \mathcal{R}^{E}$ and their covariant differentiations.

Lemma 3.1. There is a $C_{m_{0}}^{\prime}>0$ depending on $\mathcal{R}^{M}, \mathcal{R}^{E}$ and their covariant differentiations such that, for any $t \in\left[0, T_{\max }\right)$, we have

$$
\frac{d}{d t}\|V(t)\|_{H^{m_{0}}}^{2} \leqslant C_{m_{0}}^{\prime} \cdot\left(1+\left\|V_{0}\right\|_{H^{m_{0}}}^{2}\right) \cdot\left\{\|V(t)\|_{H^{m_{0}}}^{2}+\|V(t)\|_{H^{m_{0}}}^{4}\right\} .
$$

Proof. Given $1 \leqslant k \leqslant m_{0}$, we consider

$$
\begin{aligned}
& \sum_{r+q+j=k} \int_{M}\left|D^{k} V\right| \cdot\left|D^{r} V\right| \cdot\left|D^{q} V\right| \cdot\left|D^{j} V\right| d M \\
= & \sum_{\substack{r+q+j=k \\
\max \{r, q, j\}=k}} \int_{M}\left|D^{k} V\right| \cdot\left|D^{r} V\right| \cdot\left|D^{q} V\right| \cdot\left|D^{j} V\right| d M \\
& +\sum_{\substack{r+q+j=k \\
\max \{r, q, j\} \leqslant k-1}} \int_{M}\left|D^{k} V\right| \cdot\left|D^{r} V\right| \cdot\left|D^{q} V\right| \cdot\left|D^{j} V\right| d M .
\end{aligned}
$$

Clearly,

$$
\sum_{\substack{r+q+j=k \\ \max \{r, q, j\}=k}} \int_{M}\left|D^{k} V\right| \cdot\left|D^{r} V\right| \cdot\left|D^{q} V\right| \cdot\left|D^{j} V\right| d M \lesssim\left\|D^{k} V\right\|_{2}^{2} \cdot\|V\|_{\infty}^{2} \leqslant\left\|D^{k} V\right\|_{2}^{2} \cdot\left\|V_{0}\right\|_{\infty}^{2} .
$$

And we want to derive the following

$$
\begin{aligned}
& \sum_{\substack{r+q+j=k \\
\max \{r, q, j\} \leqslant k-1}} \int_{M}\left|D^{k} V\right| \cdot\left|D^{r} V\right| \cdot\left|D^{q} V\right| \cdot\left|D^{j} V\right| d M \\
& \leqslant \sum_{\substack{r+q+j=k \\
\max \{r, q, j\} \leqslant k-1}}\left\|D^{k} V\right\|_{2} \cdot\left\|D^{r} V\right\|_{p_{r}} \cdot\left\|D^{q} V\right\|_{p_{q}} \cdot\left\|D^{j} V\right\|_{p_{j}} .
\end{aligned}
$$

where $p_{r}, p_{q}$ and $p_{j}$, belonging to $[1, \infty]$, will be determined later and satisfy

$$
\frac{1}{p_{r}}+\frac{1}{p_{q}}+\frac{1}{p_{j}}=\frac{1}{2}
$$

And then we employ Theorem 2.1 due to [3] to obtain

$$
\begin{aligned}
\left\|D^{r} V\right\|_{p_{r}} \lesssim\|V\|_{H^{m_{0}}}^{a_{r}} \cdot\|V\|_{2}^{1-a_{r}} \leqslant\|V\|_{H^{m_{0}}} \\
\left\|D^{q} V\right\|_{p_{q}} \lesssim\|V\|_{H^{m_{0}}}^{a_{q}} \cdot\|V\|_{2}^{1-a_{q}} \leqslant\|V\|_{H^{m_{0}}},
\end{aligned}
$$

and

$$
\left\|D^{j} V\right\|_{p_{j}} \lesssim\|V\|_{H^{m_{0}}}^{a_{j}} \cdot\|V\|_{2}^{1-a_{j}} \leqslant\|V\|_{H^{m_{0}}}
$$


We hope $p_{r}, p_{q}$ and $p_{j}$ meet the next conditions:

\section{Condition 1.}

$$
\frac{1}{p_{r}}=\frac{r}{m}+\frac{1}{2}-a_{r} \cdot \frac{m_{0}}{m} \quad \text { with } \quad a_{r} \in\left[\frac{r}{m_{0}}, 1\right),
$$

which is equivalent to

$$
\frac{1}{p_{r}} \in\left(\frac{r-m_{0}}{m}+\frac{1}{2}, \frac{1}{2}\right]
$$

\section{Condition 2.}

$$
\frac{1}{p_{q}}=\frac{q}{m}+\frac{1}{2}-a_{q} \cdot \frac{m_{0}}{m} \quad \text { with } \quad a_{q} \in\left[\frac{q}{m_{0}}, 1\right),
$$

which is equivalent to

$$
\frac{1}{p_{q}} \in\left(\frac{q-m_{0}}{m}+\frac{1}{2}, \frac{1}{2}\right]
$$

\section{Condition 3.}

$$
\frac{1}{p_{j}}=\frac{j}{m}+\frac{1}{2}-a_{j} \cdot \frac{m_{0}}{m} \quad \text { with } \quad a_{j} \in\left[\frac{j}{m_{0}}, 1\right),
$$

which is equivalent to

$$
\frac{1}{p_{j}} \in\left(\frac{j-m_{0}}{m}+\frac{1}{2}, \frac{1}{2}\right]
$$

We claim there exist $p_{r}, p_{q}$ and $p_{j}$ which are in $[1, \infty]$ and satisfy (3.4), (3.5), (3.6) and (3.7). Obviously, that this claim holds is equivalent to

$$
\left(\frac{r-m_{0}}{m}+\frac{1}{2}\right)+\left(\frac{q-m_{0}}{m}+\frac{1}{2}\right)+\left(\frac{j-m_{0}}{m}+\frac{1}{2}\right)<\frac{1}{2} \Longleftrightarrow k<3 m_{0}-m .
$$

Since $k \leqslant m_{0}$ and $m_{0}>\frac{m}{2}$, (3.8) is true. In other words,

$$
\sum_{\substack{r+q+j=k \\ \max \{r, q, j\} \leqslant k-1}} \int_{M}\left|D^{k} V\right| \cdot\left|D^{r} V\right| \cdot\left|D^{q} V\right| \cdot\left|D^{j} V\right| d M \lesssim\left\|D^{k} V\right\|_{2} \cdot\|V\|_{H^{m_{0}}}^{3}
$$

In conclusion,

$$
\begin{aligned}
& \sum_{r+q+j=k} \int_{M}\left|D^{k} V\right| \cdot\left|D^{r} V\right| \cdot\left|D^{q} V\right| \cdot\left|D^{j} V\right| d M \\
\lesssim & \left\|D^{k} V\right\|_{2} \cdot\|V\|_{H^{m_{0}}}^{3}+\left\|D^{k} V\right\|_{2}^{2} \cdot\left\|V_{0}\right\|_{\infty}^{2} .
\end{aligned}
$$

For the other terms of (3.3), using the same methods, we get similar estimations:

\section{Estimation 1.}

$$
\begin{aligned}
& \sum_{i+j=k-1} a_{i j} \int_{M}\left|D^{k+1} V\right| \cdot\left|D^{i+1} V\right| \cdot\left|D^{j+1} V\right| d M \\
\lesssim & \left\|D^{k+1} V\right\|_{2} \cdot\left\|D^{k} V\right\|_{2} \cdot\|D V\|_{\infty}+\sum_{\substack{i+j=k-1 \\
\max \{i, j\} \leqslant k-2}} a_{i j} \cdot\left\|D^{k+1} V\right\|_{2} \cdot\left\|D^{i+1} V\right\|_{p_{i}} \cdot\left\|D^{j+1} V\right\|_{p_{j}} \\
\lesssim & \left\|D^{k+1} V\right\|_{2} \cdot\left\|D^{k} V\right\|_{2} \cdot\|D V\|_{\infty}+\left\|D^{k+1} V\right\|_{2} \cdot\|V\|_{H^{m_{0}}}^{2}
\end{aligned}
$$




$$
\lesssim\left\|D^{k+1} V\right\|_{2} \cdot\left\|D^{k} V\right\|_{2} \cdot\|V\|_{H^{m_{0}}}+\left\|D^{k+1} V\right\|_{2} \cdot\|V\|_{H^{m_{0}}}^{2}
$$

\section{Estimation 2.}

$$
\sum_{0 \leqslant r+q \leqslant k-1} \int_{M}\left|D^{k} V\right| \cdot\left|D^{r} V\right| \cdot\left|D^{q+1} V\right| d M \lesssim\left\|V_{0}\right\|_{\infty} \cdot\left\|D^{k} V\right\|_{2}^{2}+\left\|D^{k} V\right\|_{2} \cdot\|V\|_{H^{m_{0}}}^{2},
$$

\section{Estimation 3.}

$$
\sum_{i=0}^{k} \sum_{r+q=k-1} \int_{M}\left|D^{i} V\right| \cdot\left|D^{r} V\right| \cdot\left|D^{q+1} V\right| d M \lesssim\|V\|_{H^{k}} \cdot\left\|D^{k} V\right\|_{2} \cdot\left\|V_{0}\right\|_{\infty}+\|V\|_{H^{k}} \cdot\|V\|_{H^{m_{0}}}^{2} \text {. }
$$

Summing $k$ from 0 to $m_{0}$ gives

$$
\begin{aligned}
\frac{1}{2} \frac{d}{d t}\|V\|_{H^{m_{0}}}^{2} \leqslant & -\|V\|_{H^{m_{0}+1}}^{2}+L_{m_{0}} \cdot\|V\|_{H^{m_{0}+1}} \cdot\|V\|_{H^{m_{0}}}^{2} \\
& +\tilde{L}_{m_{0}} \cdot\left\{\|V\|_{H^{m_{0}}}^{2}+\left\|V_{0}\right\|_{\infty} \cdot\|V\|_{H^{m_{0}}}^{2}\right. \\
& \left.+\|V\|_{H^{m_{0}}}^{3}+\left\|V_{0}\right\|_{\infty}^{2} \cdot\|V\|_{H^{m_{0}}}^{2}+\|V\|_{H^{m_{0}}}^{4}\right\},
\end{aligned}
$$

where $L_{m_{0}}$ is universal and $\tilde{L}_{m_{0}}$ depends on $\mathcal{R}^{M}, \mathcal{R}^{E}$ and their covariant differentiations. Then the result follows easily from Young's inequality and Sobolev embedding

$$
\left\|V_{0}\right\|_{\infty} \lesssim\left\|V_{0}\right\|_{H^{m_{0}}}
$$

This completes the proof.

Consider an ODE

$$
\left\{\begin{aligned}
\frac{d f}{d t} & =C_{m_{0}}^{\prime} \cdot\left(1+\left\|V_{0}\right\|_{H^{m_{0}}}^{2}\right) \cdot\left(f+f^{2}\right) \\
f(0) & =\left\|V_{0}\right\|_{H^{m_{0}}}^{2}
\end{aligned}\right.
$$

Solving the above equation to get an expression of $f$, we know that the maximal existence time of the solution $f\left(\cdot,\left\|V_{0}\right\|_{H^{m_{0}}}\right)$ to (3.10) is not smaller than

$$
T^{*}:=\frac{1}{C_{m_{0}}^{\prime} \cdot\left(1+\left\|V_{0}\right\|_{H^{m_{0}}}^{2}\right)} \log \left(\frac{1+2\left\|V_{0}\right\|_{H^{m_{0}}}^{2}}{2\left\|V_{0}\right\|_{H^{m_{0}}}^{2}}\right)
$$

And $f\left(t,\left\|V_{0}\right\|_{H^{m_{0}}}\right)$ is monotone increasing with respect to $t$. In other words, for all $t \in\left[0, T^{*}\right]$,

$$
f\left(t,\left\|V_{0}\right\|_{H^{m_{0}}}\right) \leqslant f\left(T^{*},\left\|V_{0}\right\|_{H^{m_{0}}}\right)=1+2\left\|V_{0}\right\|_{H^{m_{0}}}^{2} .
$$

By comparison principle of ODE, we know that for any $t \in\left[0, \min \left\{T_{\max }, T^{*}\right\}\right.$ ),

$$
\|V(t)\|_{H^{m_{0}}} \leqslant \sqrt{1+2\left\|V_{0}\right\|_{H^{m_{0}}}^{2}} .
$$

In the sequel, we focus on the case that $k$ is sufficiently big.

Lemma 3.2. When $k \geqslant m_{0}+1$, there is a $Q_{k}>0$ depending on $\mathcal{R}^{M}, \mathcal{R}^{E}$ and their covariant differentiations such that, for any $t \in\left[0, T_{\max }\right)$, we have

$$
\begin{aligned}
& \frac{d}{d t}\|V(t)\|_{H^{k}}^{2}+\|V(t)\|_{H^{k+1}}^{2} \\
\leqslant & Q_{k} \cdot\left[\|V(t)\|_{H^{k}}^{2} \cdot\|V(t)\|_{H^{m_{0}}}^{2}+\|V(t)\|_{H^{k-1}}^{4}+\|V(t)\|_{H^{k}}^{2} \cdot\left(1+\left\|V_{0}\right\|_{\infty}+\left\|V_{0}\right\|_{\infty}^{2}\right)\right.
\end{aligned}
$$




$$
\left.+\|V(t)\|_{H^{k-1}}^{2} \cdot\left(\|V(t)\|_{H^{m_{0}}}^{2}+\|V(t)\|_{H^{m_{0}}}^{4}\right)+\|V(t)\|_{H^{k-1}}^{6}\right] .
$$

Proof. Firstly, let us calculate one term of (3.3). Applying the same method of (3.9), one can see easily that there are $p_{i}$ belonging to $[1, \infty]$ such that the following inequalities hold

$$
\begin{aligned}
& \sum_{i+j=k-1} a_{i j} \int_{M}\left|D^{k+1} V\right| \cdot\left|D^{i+1} V\right| \cdot\left|D^{j+1} V\right| d M \\
\lesssim & \left\|D^{k+1} V\right\|_{2} \cdot\left\|D^{k} V\right\|_{2} \cdot\|D V\|_{\infty}+\left\|D^{k+1} V\right\|_{2} \cdot\left\|D^{k-1} V\right\|_{2} \cdot\left\|D^{2} V\right\|_{\infty} \\
& +\sum_{\substack{i+j=k-1 \\
\max \{i, j\} \leqslant k-3}} a_{i j} \cdot\left\|D^{k+1} V\right\|_{2} \cdot\left\|D^{i+1} V\right\|_{p_{i}} \cdot\left\|D^{j+1} V\right\|_{p_{j}} \\
\lesssim & \left\|D^{k+1} V\right\|_{2} \cdot\left\|D^{k} V\right\|_{2} \cdot\|V\|_{H^{m_{0}}}+\left\|D^{k+1} V\right\|_{2} \cdot\left\|D^{k-1} V\right\|_{2} \cdot\|V\|_{H^{m_{0}}} \\
& +\left\|D^{k+1} V\right\|_{2} \cdot\|V\|_{H^{k-1}}^{2} .
\end{aligned}
$$

By the same procedure, we get the next estimations:

Estimation 4.

$$
\begin{aligned}
& \sum_{0 \leqslant r+q \leqslant k-1} \int_{M}\left|D^{k} V\right| \cdot\left|D^{r} V\right| \cdot\left|D^{q+1} V\right| d M \\
\lesssim & \left\|D^{k} V\right\|_{2}^{2} \cdot\left\|V_{0}\right\|_{\infty}+\left\|D^{k} V\right\|_{2} \cdot\left\|D^{k-1} V\right\|_{2} \cdot\|V\|_{H^{m_{0}}} \\
+ & \left\|D^{k} V\right\|_{2} \cdot\left\|D^{k-2} V\right\|_{2} \cdot\|V\|_{H^{m_{0}}}+\left\|D^{k} V\right\|_{2} \cdot\|V\|_{H^{k-1}}^{2}
\end{aligned}
$$

\section{Estimation 5.}

$$
\begin{aligned}
& \text { 14) } \sum_{r+q+j=k} \int_{M}\left|D^{k} V\right| \cdot\left|D^{r} V\right| \cdot\left|D^{q} V\right| \cdot\left|D^{j} V\right| d M \\
& \lesssim \int_{M}\left|D^{k} V\right|^{2} \cdot|V|^{2} d M+\int_{M}\left|D^{k} V\right| \cdot\left|D^{k-1} V\right| \cdot|D V| \cdot|V| d M \\
& +\sum_{\substack{r+q+j=k \\
\max \{r, q, j\} \leqslant k-2}} \int_{M}\left|D^{k} V\right| \cdot\left|D^{r} V\right| \cdot\left|D^{q} V\right| \cdot\left|D^{j} V\right| d M \\
& \lesssim\left\|D^{k} V\right\|_{2}^{2} \cdot\|V\|_{\infty}^{2}+\left\|D^{k} V\right\|_{2} \cdot\left\|D^{k-1} V\right\|_{2} \cdot\|D V\|_{\infty} \cdot\|V\|_{\infty}+\left\|D^{k} V\right\|_{2} \cdot\|V\|_{H^{k-1}}^{3} \\
& \lesssim\left\|D^{k} V\right\|_{2}^{2} \cdot\left\|V_{0}\right\|_{\infty}^{2}+\left\|D^{k} V\right\|_{2} \cdot\left\|D^{k-1} V\right\|_{2} \cdot\|V\|_{H^{m_{0}}}^{2}+\left\|D^{k} V\right\|_{2} \cdot\|V\|_{H^{k-1}}^{3} .
\end{aligned}
$$

\section{Estimation 6.}

$$
\begin{aligned}
& \sum_{i=0}^{k} \sum_{r+q=k-1} \int_{M}\left|D^{i} V\right| \cdot\left|D^{r} V\right| \cdot\left|D^{q+1} V\right| d M \\
\lesssim & \|V\|_{H^{k}} \cdot\|V\|_{\infty} \cdot\left\|D^{k} V\right\|_{2}+\|V\|_{H^{k}} \cdot\left\|D^{k-1} V\right\|_{2} \cdot\|D V\|_{\infty} \\
& +\|V\|_{H^{k}} \cdot\left\|D^{k-2} V\right\|_{2} \cdot\left\|D^{2} V\right\|_{\infty}+\|V\|_{H^{k}} \cdot\|V\|_{H^{k-1}}^{2} \\
\lesssim & \|V\|_{H^{k}} \cdot\left\|V_{0}\right\|_{\infty} \cdot\left\|D^{k} V\right\|_{2}+\|V\|_{H^{k}} \cdot\left\|D^{k-1} V\right\|_{2} \cdot\|V\|_{H^{m_{0}}} \\
& +\|V\|_{H^{k}} \cdot\left\|D^{k-2} V\right\|_{2} \cdot\|V\|_{H^{m_{0}}}+\|V\|_{H^{k}} \cdot\|V\|_{H^{k-1}}^{2}
\end{aligned}
$$


Substituting (3.12), (3.13), (3.14) and (3.15) into (3.3) and then summing $k$ lead to

$$
\begin{aligned}
\frac{1}{2} \frac{d}{d t}\|V\|_{H^{k}}^{2} \leqslant & -\|V\|_{H^{k+1}}^{2}+\hat{Q}_{k} \cdot\left(\|V\|_{H^{k+1}} \cdot\|V\|_{H^{k}} \cdot\|V\|_{H^{m_{0}}}+\|V\|_{H^{k+1}} \cdot\|V\|_{H^{k-1}}^{2}\right) \\
& +\tilde{Q}_{k} \cdot\left(\|V\|_{H^{k}}^{2}+\|V\|_{H^{k}}^{2} \cdot\left\|V_{0}\right\|_{\infty}+\|V\|\left\|_{H^{k}} \cdot\right\| V\left\|_{H^{k-1}} \cdot\right\| V \|_{H^{m_{0}}}\right. \\
& +\|V\|_{H^{k}} \cdot\|V\|_{H^{k-1}}^{2}+\|V\|_{H^{k}}^{2} \cdot\left\|V_{0}\right\|_{\infty}^{2}+\|V\|_{H^{k}} \cdot\|V\|_{H^{k-1}} \cdot\|V\|_{H^{m_{0}}}^{2} \\
& \left.+\|V\|_{H^{k}} \cdot\|V\|_{H^{k-1}}^{3}\right),
\end{aligned}
$$

where $\tilde{Q}_{k}>0$ depends on $\mathcal{R}^{M}, \mathcal{R}^{E}$ and their covariant differentiations. Using Young's inequality, we conclude this theorem.

Note that (3.11) is linear for $\|V\|_{H^{k}}^{2}$. It is now clear that inductively using (3.11) one can show the existence of $N_{k}=N\left(\left\|V_{0}\right\|_{H^{k}}, Q_{k}, Q_{k-1}, \cdots, Q_{m_{0}+1}\right)$ for any $k \geqslant m_{0}+1$ such that

which implies

$$
\|V(t)\|_{H^{k}} \leqslant N_{k} \quad \forall t \in\left[0, \min \left\{T_{\max }, T^{*}\right\}\right),
$$

$$
T_{\max } \geqslant T^{*}
$$

Now we return to prove Theorem 1.1. Define

$$
h(x):=\frac{1}{C_{m_{0}}^{\prime} \cdot(1+x)} \log \left(\frac{1+2 x}{2 x}\right)
$$

and we observe that it is a monotone decreasing function. Given $l \geqslant m_{0}+1$ and $V_{0} \in$ $H^{l}(E)$, there are $V_{i 0} \in \Gamma^{\infty}(E)$ such that as $i \rightarrow \infty$,

$$
V_{i 0} \longrightarrow V_{0} \quad \text { strongly in } H^{l}(E) \text {. }
$$

By the above discussion we know there exist

$$
T_{i}^{*} \geqslant h\left(\left\|V_{i 0}\right\|_{H^{m_{0}}}^{2}\right)>0 \quad \text { and } \quad V_{i} \in C^{\infty}\left(\left[0, T_{i}^{*}\right), \Gamma^{\infty}(E)\right)
$$

such that

$$
\left\{\begin{array}{l}
\partial_{t} V_{i}=\Delta V_{i}+V_{i} \times \Delta V_{i}-\lambda \cdot\left(1+\mu\left|V_{i}\right|^{2}\right) V_{i} \\
V_{i}(0, \cdot)=V_{i 0},
\end{array}\right.
$$

here $T_{i}^{*}$ is the maximal existence time of $V_{i}$. Obviously, when $i$ is enough large,

$$
\left\|V_{i 0}\right\|_{H^{m_{0}}}^{2} \leqslant\left\|V_{0}\right\|_{H^{m_{0}}}^{2}+1 \quad \text { and } \quad\left\|V_{i 0}\right\|_{H^{l}} \leqslant\left\|V_{0}\right\|_{H^{l}}+1
$$

which imply

$$
T_{i}^{*} \geqslant h\left(\left\|V_{0}\right\|_{H^{m_{0}}}^{2}+1\right):=2 \tilde{\delta}>0
$$

and

$$
\left\|V_{i}(t)\right\|_{H^{l}} \leqslant N\left(\left\|V_{0}\right\|_{H^{l}}+1, Q_{k}, Q_{k-1}, \cdots, Q_{m_{0}+1}\right) \quad \forall t \in[0, \tilde{\delta}] .
$$

Then $V_{i}$ is a bounded sequence in $L^{\infty}\left([0, \tilde{\delta}], H^{l}(E)\right)$. It is not hard to verify that $\partial_{t} V_{i}$ is a bounded sequence in $L^{\infty}\left([0, \tilde{\delta}], L^{2}(E)\right)$. So there exists a $V \in L^{\infty}\left([0, \tilde{\delta}], H^{l}(E)\right)$ and a subsequence which is still denoted by $\left\{V_{i}\right\}$ such that

$$
V_{i} \rightarrow V \quad \text { weakly } * \text { in } L^{\infty}\left([0, \tilde{\delta}], H^{l}(E)\right) .
$$


By Aubin-Lions lemma, one can find a subsequence still denoted by $\left\{V_{i}\right\}$ such that

$$
V_{i} \longrightarrow V \quad \text { strongly in } L^{\infty}\left([0, \tilde{\delta}], H^{l-1}(E)\right) .
$$

Because $l-1 \geqslant m_{0}, H^{l-1}(E)$ can be embedded into $\Gamma^{2}(E)$. In other words, $V$ is a solution to (1.6). Using LLB to transform time derivatives into spatial derivatives gives that for all $0 \leqslant j \leqslant\left[\frac{l}{\hat{m}}\right]$ and all $\alpha \leqslant l-\hat{m} j$, we have

$$
\partial_{t}^{j} D^{\alpha} V \in L^{\infty}\left([0, \tilde{\delta}], L^{2}(E)\right) .
$$

Remark 3.3. The proof of (3.17) is easy if one employs induction for $j$.

At last, since $l \geqslant\left[\frac{m}{2}\right]+4$, by the same method of Theorem 3 in [4] it is not difficult to know that the solution of (1.6) with initial data $V_{0} \in H^{l}(E)$ is unique. This completes the proof.

\section{Proof of Theorem 1.2}

Now we focus on global existence of LLB. Suppose that $V$ is the local smooth solution of (1.6). Our trick is to deduce a uniform estimation for $\|V\|_{H^{k}}$. To this goal, firstly we should get a linear Gronwall inequality.

By (3.3) and Hölder inequality, we have

$$
\begin{aligned}
\frac{1}{2} \frac{d}{d t}\left\|D^{k} V\right\|_{2}^{2} \leqslant & -\left\|D^{k+1} V\right\|_{2}^{2}+\sum_{i+j=k-1} a_{i j} \cdot\left\|D^{k+1} V\right\|_{2} \cdot\left\|\left|D^{i+1} V\right| \cdot\left|D^{j+1} V\right|\right\|_{2} \\
& +\tilde{C}_{k} \cdot\left\{\|V\|_{H^{k}}^{2}+\left\|D^{k} V\right\|_{2} \sum_{0 \leqslant r+q \leqslant k-1}\left\|\left|D^{r} V\right| \cdot\left|D^{q+1} V\right|\right\|_{2}\right. \\
& \left\|D^{k} V\right\|_{2} \sum_{r+q+j=k}\left\|\left|D^{r} V\right| \cdot\left|D^{q} V\right| \cdot\left|D^{j} V\right|\right\|_{2} \\
& \left.+\|V\|_{H^{k}} \sum_{r+q=k-1}\left\|\left|D^{r} V\right| \cdot\left|D^{q+1} V\right|\right\|_{2}\right\} .
\end{aligned}
$$

For the second term on the right hand side of (4.1),

$$
\left\|\left|D^{i+1} V\right| \cdot\left|D^{j+1} V\right|\right\|_{2} \leqslant\left\|D^{i+1} V\right\|_{\frac{2 k+2}{i+1}} \cdot\left\|D^{k-i} V\right\|_{\frac{2 k+2}{k-i}}
$$

since $i+j=k-1$. Theorem 2.4 implies

$$
\left\|D^{i+1} V\right\|_{\frac{2 k+2}{i+1}} \lesssim\left\|D^{k+1} V\right\|_{2}^{\frac{i+1}{k+1}} \cdot\|V\|_{\infty}^{\frac{k-i}{k+1}}
$$

and

$$
\left\|D^{k-i} V\right\|_{\frac{2 k+2}{k-i}} \lesssim\left\|D^{k+1} V\right\|_{2}^{\frac{k-i}{k+1}} \cdot\|V\|_{\infty}^{\frac{i+1}{k+1}}
$$

So

$$
\begin{aligned}
& \sum_{i+j=k-1} a_{i j} \cdot\left\|D^{k+1} V\right\|_{2} \cdot\left\|\left|D^{i+1} V\right| \cdot\left|D^{j+1} V\right|\right\|_{2} \\
\leqslant & B_{k} \cdot\|V\|_{\infty} \cdot\left\|D^{k+1} V\right\|_{2}^{2} \leqslant B_{k} \cdot\left\|V_{0}\right\|_{\infty} \cdot\left\|D^{k+1} V\right\|_{2}^{2}
\end{aligned}
$$


where $B_{k}$ is a universal constant. By the same way, we will get

$$
\begin{aligned}
& \sum_{0 \leqslant r+q \leqslant k-1}\left\|\left|D^{r} V\right| \cdot\left|D^{q+1} V\right|\right\|_{2} \leqslant \sum_{0 \leqslant r+q \leqslant k-1}\left\|D^{r} V\right\|_{\frac{2 r+2 q+2}{r}} \cdot\left\|D^{q+1} V\right\|_{\frac{2 r+2 q+2}{q+1}} \\
\lesssim & \sum_{0 \leqslant r+q \leqslant k-1}\left[\left\|D^{r+q+1} V\right\|_{2}^{\frac{r}{r+q+1}} \cdot\|V\|_{\infty}^{\frac{q+1}{r+q+1}}\right] \cdot\left[\left\|D^{r+q+1} V\right\|_{2}^{\frac{q+1}{r+q+1}} \cdot\|V\|_{\infty}^{\frac{r}{r+q+1}}\right] \\
= & \sum_{0 \leqslant r+q \leqslant k-1}\left\|D^{r+q+1} V\right\|_{2} \cdot\|V\|_{\infty} \lesssim\|V\|_{H^{k}} \cdot\left\|V_{0}\right\|_{\infty}
\end{aligned}
$$

and

$$
\sum_{r+q=k-1}\left\|\left|D^{r} V\right| \cdot\left|D^{q+1} V\right|\right\|_{2} \lesssim\left\|D^{k} V\right\|_{2} \cdot\left\|V_{0}\right\|_{\infty} .
$$

Moreover, Theorem 2.4 yields

$$
\begin{aligned}
& \sum_{r+q+j=k}\left\|\left|D^{r} V\right| \cdot\left|D^{q} V\right| \cdot\left|D^{j} V\right|\right\|_{2} \leqslant \sum_{r+q+j=k}\left\|D^{r} V\right\|_{\frac{2 k}{r}} \cdot\left\|D^{q} V\right\|_{\frac{2 k}{q}} \cdot\left\|D^{j} V\right\|_{\frac{2 k}{j}} \\
\lesssim & \sum_{r+q+j=k}\left(\left\|D^{k} V\right\|_{2}^{\frac{r}{k}} \cdot\|V\|_{\infty}^{\frac{q+j}{k}}\right) \cdot\left(\left\|D^{k} V\right\|_{2}^{\frac{q}{k}} \cdot\|V\|_{\infty}^{\frac{r+j}{k}}\right) \cdot\left(\left\|D^{k} V\right\|_{2}^{\frac{j}{k}} \cdot\|V\|_{\infty}^{\frac{q+r}{k}}\right) \\
\lesssim & \left\|D^{k} V\right\|_{2} \cdot\|V\|_{\infty}^{2} \leqslant\left\|D^{k} V\right\|_{2} \cdot\left\|V_{0}\right\|_{\infty}^{2} .
\end{aligned}
$$

Substituting (4.2), (4.3), (4.4) and (4.5) into (4.1) leads to

$$
\begin{aligned}
& \frac{1}{2} \frac{d}{d t}\left\|D^{k} V\right\|_{2}^{2}+\left(1-B_{k} \cdot\left\|V_{0}\right\|_{\infty}\right) \cdot\left\|D^{k+1} V\right\|_{2}^{2} \\
\leqslant & G_{k} \cdot\left\{\|V\|_{H^{k}}^{2}+\left\|D^{k} V\right\|_{2} \cdot\|V\|_{H^{k}} \cdot\left\|V_{0}\right\|_{\infty}+\left\|D^{k} V\right\|_{2}^{2} \cdot\left\|V_{0}\right\|_{\infty}^{2}\right\} \\
\leqslant & G_{k} \cdot\left(1+\left\|V_{0}\right\|_{\infty}+\left\|V_{0}\right\|_{\infty}^{2}\right) \cdot\|V\|_{H^{k}}^{2},
\end{aligned}
$$

where $G_{k}$ depends upon $\mathcal{R}^{M}, \mathcal{R}^{E}$ and their covariant differentiations. In the sequel, using Gronwall inequality gives the following theorem.

Theorem 4.1. Given $N \in \mathbb{N}$, there exists an $\tilde{B}_{N}>0$ such that if $\left\|V_{0}\right\|_{\infty} \leqslant \tilde{B}_{N}$, we will obtain

$$
\left\|D^{k} V(t)\right\|_{2}^{2}+\int_{0}^{t}\left\|D^{k+1} V(s)\right\|_{2}^{2} d s \leqslant C_{k}\left(\left\|V_{0}\right\|_{H^{k}}, \tilde{B}_{N}, t\right),
$$

provided $0 \leqslant k \leqslant N$ and $t \in\left[0, T_{\max }\right)$. Here $C_{k}(x, y, t)$ is monotone increasing with respect to $x$ and $t$.

Proof. Employ induction for $N$.

In the case $N=0$, let $\tilde{B}_{0}:=1$. Taking inner product with $V$ in (1.6) and then integrating the result over $M$, we get

$$
\frac{1}{2} \frac{d}{d t}\|V(t)\|_{2}^{2}+\|D V(t)\|_{2}^{2}+\lambda \int_{M}\left(1+\mu|V(t)|^{2}\right) \cdot|V(t)|^{2} d M=0
$$

which is equivalent to

$$
\|V(t)\|_{2}^{2}+2 \int_{0}^{t}\|D V(s)\|_{2}^{2} d s
$$




$$
+2 \lambda \int_{0}^{t} d s \int_{M}\left(1+\mu|V(s)|^{2}\right) \cdot|V(s)|^{2} d M=\left\|V_{0}\right\|_{2}^{2}
$$

Assume that for all the indices not larger than $N$, (4.7) holds. Now we consider $N+1$. Take $\tilde{B}_{N+1}:=\min \left\{\tilde{B}_{N}, \frac{1}{2 B_{N+1}}\right\}$. If $\left\|V_{0}\right\|_{\infty} \leqslant \tilde{B}_{N+1}$, (4.6) gives

$$
\begin{aligned}
& \frac{1}{2} \frac{d}{d t}\left\|D^{N+1} V\right\|_{2}^{2}+\frac{1}{2}\left\|D^{N+2} V\right\|_{2}^{2} \\
\leqslant & G_{N+1} \cdot\left(1+\tilde{B}_{N+1}+\tilde{B}_{N+1}^{2}\right) \cdot\left\{\left\|D^{N+1} V\right\|_{2}^{2}+\sum_{k=0}^{N} C_{k}\left(\left\|V_{0}\right\|_{H^{k}}, \tilde{B}_{N}, t\right)\right\} .
\end{aligned}
$$

Then this theorem follows easily from Gronwall inequality. This completes the proof.

Now we return to prove Theorem [1.2. Given $T>0$ and $N \geqslant m_{0}+1=\left[\frac{m}{2}\right]+4$, we take any $V_{0} \in H^{N}(E)$ with $\left\|V_{0}\right\|_{\infty} \leqslant \frac{1}{2} \tilde{B}_{N}:=\hat{B}_{N}$ (This $\tilde{B}_{N}$ is from Theorem 4.1). Then there are $V_{0 i} \in \Gamma^{\infty}(E)$ converging to $V_{0}$ strongly in $H^{N}(E)$.

Suppose $V_{i}$ satisfies

$$
\left\{\begin{array}{l}
\partial_{t} V_{i}=\Delta V_{i}+V_{i} \times \Delta V_{i}-\lambda \cdot\left(1+\mu \cdot\left|V_{i}\right|^{2}\right) V_{i} \\
V_{i}(0, \cdot)=V_{0 i}
\end{array}\right.
$$

and its maximal existence time is $T_{i}^{*}$. As $i$ is large enough, we have

$$
\left\|V_{0 i}\right\|_{\infty} \leqslant 2\left\|V_{0}\right\|_{\infty} \leqslant \tilde{B}_{N}, \quad\left\|V_{0 i}\right\|_{H^{m_{0}}} \leqslant 2\left\|V_{0}\right\|_{H^{m_{0}}}
$$

and $\left\|V_{0 i}\right\|_{H^{N}} \leqslant 2\left\|V_{0}\right\|_{H^{N}}$.

If $T_{i}^{*}<T$, then by Theorem 4.1,

$$
\left\|V_{i}(t)\right\|_{H^{m_{0}}}^{2}+\int_{0}^{t}\left\|D V_{i}(s)\right\|_{H^{m_{0}}}^{2} d s \leqslant C_{m_{0}}\left(\left\|V_{0 i}\right\|_{H^{m_{0}}}, \tilde{B}_{N}, t\right) \leqslant C_{m_{0}}\left(2\left\|V_{0}\right\|_{H^{m_{0}}}, \tilde{B}_{N}, T\right),
$$

provided $t \in\left[0, T_{i}^{*}\right)$. Review that in the proof of Theorem 1.1 we have defined a monotone decreasing function

$$
h(x):=\frac{1}{C_{m_{0}}^{\prime} \cdot(1+x)} \log \left(\frac{1+2 x}{2 x}\right) .
$$

So for arbitrary $t \in\left[0, T_{i}^{*}\right)$, it is obvious to see

$$
h\left(\left\|V_{i}(t)\right\|_{H^{m_{0}}}^{2}\right) \geqslant h\left(C_{m_{0}}\left(2\left\|V_{0}\right\|_{H^{m_{0}}}, \tilde{B}_{N}, T\right)\right):=\delta_{0}>0 .
$$

Now we bring in a new system

$$
\left\{\begin{array}{l}
\partial_{t} \hat{V}_{i}=\Delta \hat{V}_{i}+\hat{V}_{i} \times \Delta \hat{V}_{i}-\lambda \cdot\left(1+\mu \cdot\left|\hat{V}_{i}\right|^{2}\right) \hat{V}_{i} \quad \text { in } \quad\left(T_{i}^{*}-\frac{\delta_{0}}{2}, \infty\right) \times M \\
\hat{V}_{i}\left(T_{i}^{*}-\frac{\delta_{0}}{2}, \cdot\right)=V_{i}\left(T_{i}^{*}-\frac{\delta_{0}}{2}, \cdot\right)
\end{array}\right.
$$

The maximal existence time of $\hat{V}_{i}$ is not smaller than $h\left(\left\|V_{i}\left(T_{i}^{*}-\frac{\delta_{0}}{2}\right)\right\|_{H^{m_{0}}}^{2}\right)$ which is not smaller than $\delta_{0}$. By the uniqueness we know that for any $t \in\left[T_{i}^{*}-\frac{\delta_{0}}{2}, T_{i}^{*}\right), \hat{V}_{i}(t)=V_{i}(t)$. 
It means that $V_{i}$ can be extended to $\left[0, T_{i}^{*}+\frac{\delta_{0}}{2}\right)$. Because $T_{i}^{*}$ is maximal, we get a contradiction. So $V_{i} \in C^{\infty}\left([0, T], \Gamma^{\infty}(E)\right)$ and for all $t \in[0, T]$,

$$
\left\|V_{i}(t)\right\|_{H^{N}}^{2}+\int_{0}^{t}\left\|D V_{i}(s)\right\|_{H^{N}}^{2} d s \leqslant C_{N}\left(2\left\|V_{0}\right\|_{H^{N}}, \tilde{B}_{N}, T\right) .
$$

By the same method we prove local well-posedness one can know there is a

$$
V \in L^{\infty}\left([0, T], H^{N}(E)\right) \cap L^{2}\left([0, T], H^{N+1}(E)\right)
$$

such that $V_{i}$ converges to $V$ strongly in $L^{\infty}\left([0, T], H^{N-1}(E)\right)$ (in the sense of picking subsequence). It means $V$ is a solution of LLB.

At last, we claim (1.8) and (1.9) are true. Since (1.8) is easy, we only prove (1.9).

Proof. Employ induction for $i$.

When $i=0$, (1.9) holds.

Suppose that for all the indices not bigger than $i,(1.9)$ is true.

Now we consider $i+1$. Choose any $\beta \in[0, N+1-(\hat{m}+1)(i+1)] \cap \mathbb{Z}$. Applying $\partial_{t}^{i} D^{\beta}$ to both sides of (1.6), we get

$$
\partial_{t}^{i+1} D^{\beta} V=\partial_{t}^{i} D^{\beta} \Delta V-\partial_{t}^{i} D^{\beta}(V \times \Delta V)-\lambda \cdot \partial_{t}^{i} D^{\beta}\left[\left(1+\mu|V|^{2}\right) V\right],
$$

which implies

$$
\begin{aligned}
& \int_{0}^{T}\left\|\partial_{t}^{i+1} D^{\beta} V(s)\right\|_{2}^{2} d s \\
\lesssim & \int_{0}^{T}\left\|\partial_{t}^{i} D^{\beta+2} V(s)\right\|_{2}^{2} d s+\int_{0}^{T}\left\|\partial_{t}^{i} D^{\beta} V(s)\right\|_{2}^{2} d s \\
+ & \sum_{i^{\prime}, \beta^{\prime}} \int_{0}^{T}\left\|\left|\partial_{t}^{i^{\prime}} D^{\beta^{\prime}} V(s)\right| \cdot\left|\partial_{t}^{i-i^{\prime}} D^{\beta-\beta^{\prime}+2} V(s)\right|\right\|_{2}^{2} d s \\
+ & \sum_{\substack{i_{1}+i_{2}+i_{3}=i \\
\beta_{1}+\beta_{2}+\beta_{3}=\beta}} \int_{0}^{T}\left\|\left|\partial_{t}^{i_{1}} D^{\beta_{1}} V(s)\right| \cdot\left|\partial_{t}^{i_{2}} D^{\beta_{2}} V(s)\right| \cdot\left|\partial_{t}^{i_{3}} D^{\beta_{3}} V(s)\right|\right\|_{2}^{2} d s .
\end{aligned}
$$

Because $\hat{m}:=\max \left\{2,\left[\frac{m}{2}\right]+1\right\}$,

$i^{\prime} \leqslant i \leqslant\left[\frac{N+1}{\hat{m}+1}\right] \leqslant\left[\frac{N}{\hat{m}}\right] \quad$ and $\quad \beta^{\prime} \leqslant \beta \leqslant N+1-(\hat{m}+1)(i+1) \leqslant N-\hat{m} \cdot i^{\prime}-\hat{m}$,

(1.8) yields

$$
\left\|\partial_{t}^{i^{\prime}} D^{\beta^{\prime}} V(t)\right\|_{\infty} \leqslant\left\|\partial_{t}^{i^{\prime}} D^{\beta^{\prime}} V(t)\right\|_{H^{\hat{m}}}<\infty \quad \forall t \in[0, T] .
$$

And since

$\hat{m}\left(i-i^{\prime}\right)+\left(\beta-\beta^{\prime}+2\right) \leqslant \hat{m} \cdot i+\beta+2 \leqslant \hat{m} \cdot i+N+1-(\hat{m}+1)(i+1)+2=N-i-\hat{m}+2 \leqslant N-i \leqslant N$, by (1.8) we have

$$
\int_{0}^{T}\left\|\partial_{t}^{i-i^{\prime}} D^{\beta-\beta^{\prime}+2} V(s)\right\|_{2}^{2} d s \leqslant \sup _{t \in[0, T]}\left\{\left\|\partial_{t}^{i-i^{\prime}} D^{\beta-\beta^{\prime}+2} V(t)\right\|_{2}^{2}\right\} \cdot T<\infty .
$$


So

$$
\begin{aligned}
& \int_{0}^{T}\left\|\left|\partial_{t}^{i^{\prime}} D^{\beta^{\prime}} V(s)\right| \cdot\left|\partial_{t}^{i-i^{\prime}} D^{\beta-\beta^{\prime}+2} V(s)\right|\right\|_{2}^{2} d s \\
\leqslant & \sup _{t \in[0, T]}\left\{\left\|\partial_{t}^{i^{\prime}} D^{\beta^{\prime}} V(t)\right\|_{\infty}^{2}\right\} \cdot \int_{0}^{T}\left\|\partial_{t}^{i-i^{\prime}} D^{\beta-\beta^{\prime}+2} V(s)\right\|_{2}^{2} d s<\infty
\end{aligned}
$$

For the other terms on the right hand side of (4.12), using similar method we know all of them are strictly smaller than $\infty$.

This completes the proof.

\section{Proof of Theorem 1.3}

In this section, we need some formulas. Their proofs are tedious. So we only list the results.

Formula 3. Suppose that $V \in \Gamma^{2}(E)$. Then we will obtain

$$
\begin{aligned}
\|\Delta V\|_{2}^{2}= & \left\|D^{2} V\right\|_{2}^{2}+2 \int_{M}\left\langle D V, D V * \mathcal{R}^{E}\right\rangle d M \\
& +\int_{M}\left\langle D V, V * D \mathcal{R}^{E}\right\rangle d M+\int_{M}\left\langle D V, D V * \mathcal{R}^{M}\right\rangle d M .
\end{aligned}
$$

Remark 5.1. Formula 3 easily implies

$$
\left\|D^{2} V\right\|_{2}^{2} \leqslant\|\Delta V\|_{2}^{2}+\eta \cdot\left(\|D V\|_{2}^{2}+\|V\|_{2}^{2}\right),
$$

where $\eta$ depends on $\mathcal{R}^{M}, \mathcal{R}^{E}$ and their covariant derivatives.

Formula 4. Given $V \in \Gamma^{3}(E)$,

$$
\begin{aligned}
\|\Delta D V\|_{2}^{2}= & \left\|D^{3} V\right\|_{2}^{2}+3 \int_{M}\left\langle D^{2} V, D^{2} V * \mathcal{R}^{M}\right\rangle d M+2 \int_{M}\left\langle D^{2} V, D^{2} V * \mathcal{R}^{E}\right\rangle d M \\
& +\int_{M}\left\langle D^{2} V, D V * \nabla \mathcal{R}^{M}\right\rangle d M+\int_{M}\left\langle D^{2} V, D V * D \mathcal{R}^{E}\right\rangle d M .
\end{aligned}
$$

Remark 5.2. From Formula 4 it follows that

$$
\left\|D^{3} V\right\|_{2}^{2} \leqslant\|\Delta D V\|_{2}^{2}+\eta_{2} \cdot\left(\left\|D^{2} V\right\|_{2}^{2}+\|D V\|_{2}^{2}\right),
$$

where $\eta_{2}$ depends on $\mathcal{R}^{M}, \mathcal{R}^{E}$ and their covariant derivatives. Since by (2.9) we have

$$
\Delta D V=D \Delta V+\mathfrak{q}_{1}\left(V, \mathcal{R}^{E}\right)+\mathfrak{q}_{0}\left(D V, \mathcal{R}^{M}\right)
$$

integration by parts and Hölder's inequality yield

$$
\|\Delta D V\|_{2}^{2} \leqslant\|D \Delta V\|_{2}^{2}+\eta_{3} \cdot\left(\left\|D^{2} V\right\|_{2}^{2}+\|D V\|_{2}^{2}+\|V\|_{2}^{2}\right)
$$

where $\eta_{3}$ depends on $\mathcal{R}^{M}, \mathcal{R}^{E}$ and their covariant derivatives. Substituting (5.3) into (5.2) gives

$$
\left\|D^{3} V\right\|_{2}^{2} \leqslant\|D \Delta V\|_{2}^{2}+\left(\eta_{2}+\eta_{3}\right) \cdot\left(\left\|D^{2} V\right\|_{2}^{2}+\|D V\|_{2}^{2}+\|V\|_{2}^{2}\right) .
$$


Formula 5. If $V \in \Gamma^{4}(E)$, then

$$
\begin{aligned}
\left\|D^{4} V\right\|_{2}^{2}= & \left\|\Delta^{2} V\right\|_{2}^{2}+\int_{M}\left\langle\mathfrak{q}_{3}\left(V, \mathcal{R}^{E}\right), D^{3} V\right\rangle d M+\int_{M}\left\langle\mathfrak{q}_{2}\left(D V, \mathcal{R}^{M}\right), D^{3} V\right\rangle d M \\
& +\int_{M}\left\langle\mathfrak{q}_{1}\left(D V, \mathcal{R}^{E}\right), \mathfrak{q}_{1}\left(D V, \mathcal{R}^{E}\right)\right\rangle d M+\int_{M}\left\langle\mathfrak{q}_{1}\left(D V, \mathcal{R}^{E}\right), \mathfrak{q}_{1}\left(D V, \mathcal{R}^{M}\right)\right\rangle d M \\
& +\int_{M}\left\langle\mathfrak{q}_{1}\left(D V, \mathcal{R}^{M}\right), \mathfrak{q}_{1}\left(D V, \mathcal{R}^{M}\right)\right\rangle d M .
\end{aligned}
$$

Remark 5.3. Formula 5, Hölder's inequality and Young's inequality lead to

$$
\begin{aligned}
\left\|D^{4} V\right\|_{2}^{2} & \leqslant\left\|\Delta^{2} V\right\|_{2}^{2}+\eta_{5} \cdot\left(\left\|D^{3} V\right\|_{2}^{2}+\left\|D^{2} V\right\|_{2}^{2}+\|D V\|_{2}^{2}+\|V\|_{2}^{2}\right) \\
& \leqslant\left\|\Delta^{2} V\right\|_{2}^{2}+\eta_{6} \cdot\left(\|D \Delta V\|_{2}^{2}+\|\Delta V\|_{2}^{2}+\|D V\|_{2}^{2}+\|V\|_{2}^{2}\right)
\end{aligned}
$$

where we have used (5.1), (5.4) and $\eta_{5}, \eta_{6}$ depend on $\mathcal{R}^{M}, \mathcal{R}^{E}$ and their covariant derivatives.

Formula 6. Assume that $V \in \Gamma^{4}(E)$. Then we get

$$
\begin{aligned}
\left\|D^{2} \Delta V\right\|_{2}^{2} & =\left\|\Delta^{2} V\right\|_{2}^{2}+\int_{M}\left\langle\mathfrak{q}_{1}\left(\Delta V, \mathcal{R}^{E}\right), D \Delta V\right\rangle d M+\int_{M}\left\langle\mathfrak{q}_{0}\left(D \Delta V, \mathcal{R}^{M}\right), D \Delta V\right\rangle d M \\
(5.6) & \lesssim\left\|\Delta^{2} V\right\|_{2}^{2}+\eta_{8} \cdot\left(\|D \Delta V\|_{2}^{2}+\|\Delta V\|_{2}^{2}\right),
\end{aligned}
$$

where we have applied Hölder's inequality, Young's inequality and $\eta_{8}$ depends on $\mathcal{R}^{M}$, $\mathcal{R}^{E}$ and their covariant derivatives.

Now let us go on to prove Theorem 1.3. Suppose that $V \in C^{\infty}\left(\left[0, T^{*}\right), \Gamma^{\infty}(E)\right)$ is the unique local smooth solution of (1.6), where $T^{*}$ is its maximal existence time. First of all, we shall estimate $\|D V(t)\|_{\infty}$ for all $t \in\left[0, T^{*}\right)$. By Sobolev embedding it is easy to see that we only need to get a uniform upper bound of $\|D V(t)\|_{H^{2}}$ (Note that in this section $m=2)$. Combining (5.1) and (5.4) one can know that we only need to estimate

$$
\|D V(t)\|_{2}^{2}+\|\Delta V(t)\|_{2}^{2}+\|D \Delta V(t)\|_{2}^{2} .
$$

Using the same method of (2.2) in [4] we can get

$$
\|D V(t)\|_{2}^{2}+\int_{0}^{t}\|\Delta V(s)\|_{2}^{2} d s \leqslant \lambda^{2} \cdot\left(1+\mu\left\|V_{0}\right\|_{\infty}^{2}\right)^{2} \cdot\left\|V_{0}\right\|_{2}^{2} \cdot t+\left\|D V_{0}\right\|_{2}^{2}
$$

For $\|\Delta V(t)\|_{2}^{2}$, our trick is to deduce a Gronwall's inequality. (1.6) yields

$$
\begin{aligned}
& \frac{1}{2} \frac{d}{d t}\|\Delta V(t)\|_{2}^{2}+\int_{M}|D \Delta V(t)|^{2} d M+\lambda \cdot\|\Delta V(t)\|_{2}^{2} \\
= & -\int_{M}[D V(t) \times \Delta V(t)] * D \Delta V(t) d M-\lambda \mu \cdot \int_{M}\left\langle\Delta\left[|V(t)|^{2} \cdot V(t)\right], \Delta V(t)\right\rangle d M \\
\leqslant & \int_{M}|D V(t)| \cdot|\Delta V(t)| \cdot|D \Delta V(t)| d M+C \cdot\|V(t)\|_{\infty}^{2} \cdot\left(\|\Delta V(t)\|_{2}^{2}+\|D V(t)\|_{4}^{2}\right) \\
\leqslant & \|D V(t)\|_{4} \cdot\|\Delta V(t)\|_{4} \cdot\|D \Delta V(t)\|_{2}+C \cdot\|V(t)\|_{\infty}^{2} \cdot\left(\|\Delta V(t)\|_{2}^{2}+\|D V(t)\|_{4}^{2}\right),
\end{aligned}
$$


here $C$ is a universal constant. Theorem 2.1 of [3], (5.1) and (5.4) give

$$
\begin{aligned}
\|D V(t)\|_{4} & \lesssim\|D V(t)\|_{H^{2}}^{\frac{1}{4}} \cdot\|D V(t)\|_{2}^{\frac{3}{4}} \\
(5.9) & \leqslant \eta_{4} \cdot\left(\|D \Delta V(t)\|_{2}^{\frac{1}{4}}+\|\Delta V(t)\|_{2}^{\frac{1}{4}}+\|D V(t)\|_{2}^{\frac{1}{4}}+\|V(t)\|_{2}^{\frac{1}{4}}\right) \cdot\|D V(t)\|_{2}^{\frac{3}{4}}
\end{aligned}
$$

and

$$
\begin{aligned}
\|\Delta V(t)\|_{4} & \lesssim\|\Delta V(t)\|_{H^{1}}^{\frac{1}{2}} \cdot\|\Delta V(t)\|_{2}^{\frac{1}{2}} \\
& \lesssim\left(\|D \Delta V(t)\|_{2}^{\frac{1}{2}}+\|\Delta V(t)\|_{2}^{\frac{1}{2}}\right) \cdot\|\Delta V(t)\|_{2}^{\frac{1}{2}}
\end{aligned}
$$

where $\eta_{4}$ depends upon $\eta_{2}, \eta_{3}$ and $\eta$. Thus we derive

$$
\begin{aligned}
& \|D V(t)\|_{4} \cdot\|\Delta V(t)\|_{4} \cdot\|D \Delta V(t)\|_{2} \\
\lesssim & \eta_{4} \cdot\|D V(t)\|_{2}^{\frac{3}{4}} \cdot\|\Delta V(t)\|_{2}^{\frac{1}{2}} \cdot\|D \Delta V(t)\|_{2}^{\frac{7}{4}} \\
& +\eta_{4} \cdot\|D V(t)\|_{2}^{\frac{3}{4}} \cdot\|\Delta V(t)\|_{2}^{\frac{3}{4}} \cdot\|D \Delta V(t)\|_{2}^{\frac{3}{2}} \\
& +\eta_{4} \cdot\|D V(t)\|_{2} \cdot\|\Delta V(t)\|_{2}^{\frac{1}{2}} \cdot\|D \Delta V(t)\|_{2}^{\frac{3}{2}} \\
& +\eta_{4} \cdot\|D V(t)\|_{2}^{\frac{3}{4}} \cdot\|\Delta V(t)\|_{2} \cdot\|D \Delta V(t)\|_{2}^{\frac{5}{4}} \\
& +\eta_{4} \cdot\|D V(t)\|_{2}^{\frac{3}{4}} \cdot\|\Delta V(t)\|_{2}^{\frac{5}{4}} \cdot\|D \Delta V(t)\|_{2} \\
& +\eta_{4} \cdot\|D V(t)\|_{2} \cdot\|\Delta V(t)\|_{2} \cdot\|D \Delta V(t)\|_{2} \\
& +\eta_{4} \cdot\|V(t)\|_{2}^{\frac{1}{4}} \cdot\|D V(t)\|_{2}^{\frac{3}{4}} \cdot\|\Delta V(t)\|_{2}^{\frac{1}{2}} \cdot\|D \Delta V(t)\|_{2}^{\frac{3}{2}} \\
& +\eta_{4} \cdot\|V(t)\|_{2}^{\frac{1}{4}} \cdot\|D V(t)\|_{2}^{\frac{3}{4}} \cdot\|\Delta V(t)\|_{2} \cdot\|D \Delta V(t)\|_{2} \\
\leqslant & \eta_{4} \gamma_{1} \cdot\left(\|\Delta V(t)\|_{2}^{\frac{1}{2}} \cdot\|D \Delta V(t)\|_{2}^{\frac{7}{4}}+\|\Delta V(t)\|_{2}^{\frac{3}{4}} \cdot\|D \Delta V(t)\|_{2}^{\frac{3}{2}}\right. \\
& +\|\Delta V(t)\|_{2}^{\frac{1}{2}} \cdot\|D \Delta V(t)\|_{2}^{\frac{3}{2}}+\|\Delta V(t)\|_{2} \cdot\|D \Delta V(t)\|_{2}^{\frac{5}{4}} \\
& \left.+\|\Delta V(t)\|_{2}^{\frac{5}{4}} \cdot\|D \Delta V(t)\|_{2}+\|\Delta V(t)\|_{2} \cdot\|D \Delta V(t)\|_{2}\right),
\end{aligned}
$$

where $\gamma_{1}$ relies on $\left\|V_{0}\right\|_{\infty},\left\|V_{0}\right\|_{2},\left\|D V_{0}\right\|_{2}$ and $t$. From Young's inequality it follows that

$$
\begin{gathered}
\|D V(t)\|_{4} \cdot\|\Delta V(t)\|_{4} \cdot\|D \Delta V(t)\|_{2} \\
\leqslant \gamma_{2} \cdot\left(\|\Delta V(t)\|_{2}^{4}+1\right)+\frac{1}{4} \cdot\|D \Delta V(t)\|_{2}^{2},
\end{gathered}
$$

where $\gamma_{2}$ is dependent of $\mathcal{R}^{M}, \mathcal{R}^{E}$ and their covariant derivatives, $\left\|V_{0}\right\|_{\infty},\left\|V_{0}\right\|_{2},\left\|D V_{0}\right\|_{2}$ and $t$. By the same way, we have

$$
\begin{aligned}
& \|V(t)\|_{\infty}^{2} \cdot\left(\|\Delta V(t)\|_{2}^{2}+\|D V(t)\|_{4}^{2}\right) \\
\lesssim & \|V(t)\|_{\infty}^{2} \cdot\|\Delta V(t)\|_{2}^{2} \\
& +\eta_{4}^{2} \cdot\left\|V_{0}\right\|_{\infty}^{2} \cdot\left(\|D \Delta V(t)\|_{2}^{\frac{1}{2}}+\|\Delta V(t)\|_{2}^{\frac{1}{2}}+\|D V(t)\|_{2}^{\frac{1}{2}}+\|V(t)\|_{2}^{\frac{1}{2}}\right) \cdot\|D V(t)\|_{2}^{\frac{3}{2}} \\
\leqslant & \gamma_{3} \cdot\left(\|\Delta V(t)\|_{2}^{2}+1\right)+\frac{1}{4}\|D \Delta V(t)\|_{2}^{2},
\end{aligned}
$$


where $\gamma_{3}$ is dependent of $\mathcal{R}^{M}, \mathcal{R}^{E}$ and their covariant derivatives, $\left\|V_{0}\right\|_{\infty},\left\|V_{0}\right\|_{2},\left\|D V_{0}\right\|_{2}$ and $t$. Substituting (5.11) and (5.12) into (5.8) and Young's inequality lead to

$$
\frac{1}{2} \frac{d}{d t}\|\Delta V(t)\|_{2}^{2}+\frac{1}{2}\|D \Delta V(t)\|_{2}^{2}+\lambda\|\Delta V(t)\|_{2}^{2} \leqslant \gamma_{4} \cdot\left(\|\Delta V(t)\|_{2}^{4}+1\right),
$$

here $\gamma_{4}$ relies on $\gamma_{2}$ and $\gamma_{3}$. The generalized Gronwall's inequality says that if

$$
\frac{d f}{d t} \leqslant C \cdot f \cdot g+C
$$

then

$$
f \leqslant C \cdot \exp \left(\int_{0}^{t} g(s) d s\right)+C .
$$

So if we replace $f$ and $g$ by $\|\Delta V(t)\|_{2}^{2}$ and note that (5.7) implies the boundedness of $\int_{0}^{t} g(s) d s$, then

$$
\|\Delta V(t)\|_{2}^{2} \leqslant \gamma_{5}
$$

which implies

$$
\int_{0}^{t}\|D \Delta V(s)\|_{2}^{2} d s \leqslant 2 \gamma_{4} \cdot\left(\gamma_{5}^{2}+1\right) \cdot t,
$$

where $\gamma_{5}$ is dependent of $\mathcal{R}^{M}, \mathcal{R}^{E}$ and their covariant derivatives, $\left\|V_{0}\right\|_{\infty},\left\|V_{0}\right\|_{2},\left\|D V_{0}\right\|_{2}$, $\left\|\Delta V_{0}\right\|_{2}$ and $t$.

In the sequel, we are going to estimate $\|D \Delta V(t)\|_{2}$ for all $t \in\left[0, T^{*}\right)$. (1.6) gives

$$
\begin{aligned}
\frac{1}{2} \frac{d}{d t}\|D \Delta V(t)\|_{2}^{2}= & -\left\|\Delta^{2} V(t)\right\|_{2}^{2}-\int_{M}\left\langle\Delta^{2} V(t), \Delta[V(t) \times \Delta V(t)]\right\rangle d M \\
& +\lambda \int_{M}\left\langle\Delta\left[\left(1+\mu|V(t)|^{2}\right) \cdot V(t)\right], \Delta^{2} V(t)\right\rangle d M \\
= & -\left\|\Delta^{2} V(t)\right\|_{2}^{2}-2 \int_{M} \Delta^{2} V(t) *[D V(t) \times D \Delta V(t)] d M \\
& +\lambda \int_{M}\left\langle\Delta\left[\left(1+\mu|V(t)|^{2}\right) \cdot V(t)\right], \Delta^{2} V(t)\right\rangle d M \\
= & -\left\|\Delta^{2} V(t)\right\|_{2}^{2}-2 \int_{M} \Delta^{2} V(t) *[D V(t) \times D \Delta V(t)] d M \\
& +\lambda \mu \int_{M}\left\langle\Delta\left[|V(t)|^{2} \cdot V(t)\right], \Delta^{2} V(t)\right\rangle d M-\lambda\|D \Delta V(t)\|_{2}^{2} .
\end{aligned}
$$

On the other hand, Hölder inequality yields

$$
\begin{aligned}
& \left|\int_{M} \Delta^{2} V(t) *[D V(t) \times D \Delta V(t)] d M\right| \\
\leqslant & \|D V(t)\|_{\frac{16}{5}} \cdot\|D \Delta V(t)\|_{\frac{16}{3}} \cdot\left\|\Delta^{2} V(t)\right\|_{2}
\end{aligned}
$$

and

$$
\left|\int_{M}\left\langle\Delta\left[|V(t)|^{2} \cdot V(t)\right], \Delta^{2} V(t)\right\rangle d M\right|
$$




$$
\lesssim\|V(t)\|_{\infty}^{2} \cdot\left\|\Delta^{2} V(t)\right\|_{2} \cdot\|\Delta V(t)\|_{2}+\|V(t)\|_{\infty} \cdot\left\|\Delta^{2} V(t)\right\|_{2} \cdot\|D V(t)\|_{4}^{2}
$$

By Sobolev Embedding, we have

$$
\|D V(t)\|_{\frac{16}{5}} \lesssim\|D V(t)\|_{H^{3}}^{\frac{1}{8}} \cdot\|D V(t)\|_{2}^{\frac{7}{8}}
$$

Combining (5.1), (5.4) and (5.5) we arrive at

$$
\begin{aligned}
\|D V(t)\|_{H^{3}}^{\frac{1}{8}} \leqslant & \eta_{7} \cdot\left(\left\|\Delta^{2} V(t)\right\|_{2}^{\frac{1}{8}}+\|D \Delta V(t)\|_{2}^{\frac{1}{8}}\right. \\
& \left.+\|\Delta V(t)\|_{2}^{\frac{1}{8}}+\|D V(t)\|_{2}^{\frac{1}{8}}+\|V(t)\|_{2}^{\frac{1}{8}}\right),
\end{aligned}
$$

where $\eta_{7}$ is dependent of $\mathcal{R}^{M}, \mathcal{R}^{E}$ and their covariant derivatives. Moreover,

$$
\|D \Delta V(t)\|_{\frac{16}{3}} \lesssim\|D \Delta V(t)\|_{H^{1}}^{\frac{5}{8}} \cdot\|D \Delta V(t)\|_{2}^{\frac{3}{8}}
$$

and

$$
\|D \Delta V(t)\|_{H^{1}} \lesssim\left\|D^{2} \Delta V(t)\right\|_{2}+\|D \Delta V(t)\|_{2}
$$

By (5.6) we are led to

$$
\|D \Delta V(t)\|_{H^{1}} \lesssim\left\|\Delta^{2} V(t)\right\|_{2}+\left(\sqrt{\eta_{8}}+1\right) \cdot\|D \Delta V(t)\|_{2}+\sqrt{\eta_{8}} \cdot\|\Delta V(t)\|_{2},
$$

which implies

$$
\begin{aligned}
\|D \Delta V(t)\|_{\frac{16}{3}} \lesssim & {\left[\left\|\Delta^{2} V(t)\right\|_{2}^{\frac{5}{8}}+\left(\eta_{8}^{\frac{5}{16}}+1\right) \cdot\|D \Delta V(t)\|_{2}^{\frac{5}{8}}\right.} \\
& \left.+\eta_{8}^{\frac{5}{16}} \cdot\|\Delta V(t)\|_{2}^{\frac{5}{8}}\right] \cdot\|D \Delta V(t)\|_{2}^{\frac{3}{8}} .
\end{aligned}
$$

Furthermore, substituting (5.16), (5.17) and (5.18) into (5.15) we arrive at

$$
\begin{aligned}
& \left|\int_{M} \Delta^{2} V(t) *[D V(t) \times D \Delta V(t)] d M\right| \\
\lesssim & \eta_{7} \cdot\left(\left\|\Delta^{2} V(t)\right\|_{2}^{\frac{1}{8}}+\|D \Delta V(t)\|_{2}^{\frac{1}{8}}+\|\Delta V(t)\|_{2}^{\frac{1}{8}}+\|D V(t)\|_{2}^{\frac{1}{8}}+\|V(t)\|_{2}^{\frac{1}{8}}\right) \\
\cdot & {\left[\left\|\Delta^{2} V(t)\right\|_{2}^{\frac{5}{8}}+\left(\eta_{8}^{\frac{5}{16}}+1\right) \cdot\|D \Delta V(t)\|_{2}^{\frac{5}{8}}+\eta_{8}^{\frac{5}{16}} \cdot\|\Delta V(t)\|_{2}^{\frac{5}{8}}\right] } \\
& \cdot\|D \Delta V(t)\|_{2}^{\frac{3}{8}} \cdot\left\|\Delta^{2} V(t)\right\|_{2} \cdot\|D V(t)\|_{2}^{\frac{7}{8}} \cdot
\end{aligned}
$$

Substituting the upper bounds of $\|V(t)\|_{2},\|D V(t)\|_{2}$ and $\|\Delta V(t)\|_{2}$ into (5.19) leads to

$$
\begin{aligned}
& \left|\int_{M} \Delta^{2} V(t) *[D V(t) \times D \Delta V(t)] d M\right| \\
\leqslant & \eta_{9} \cdot\left(\left\|\Delta^{2} V(t)\right\|_{2}^{\frac{1}{8}}+\|D \Delta V(t)\|_{2}^{\frac{1}{8}}+1\right) \\
& \cdot\left(\left\|\Delta^{2} V(t)\right\|_{2}^{\frac{5}{8}}+\|D \Delta V(t)\|_{2}^{\frac{5}{8}}+1\right) \cdot\|D \Delta V(t)\|_{2}^{\frac{3}{8}} \cdot\left\|\Delta^{2} V(t)\right\|_{2} \\
\lesssim & \eta_{9} \cdot\left(\left\|\Delta^{2} V(t)\right\|_{2}^{\frac{3}{4}}+\|D \Delta V(t)\|_{2}^{\frac{3}{4}}+1\right) \cdot\|D \Delta V(t)\|_{2}^{\frac{3}{8}} \cdot\left\|\Delta^{2} V(t)\right\|_{2} \\
\leqslant & \eta_{9} \cdot\left\|\Delta^{2} V(t)\right\|_{2}^{\frac{7}{4}} \cdot\|D \Delta V(t)\|_{2}^{\frac{3}{8}}+\eta_{9} \cdot\left\|\Delta^{2} V(t)\right\|_{2} \cdot\|D \Delta V(t)\|_{2}^{\frac{9}{8}} \\
& +\eta_{9} \cdot\left\|\Delta^{2} V(t)\right\|_{2} \cdot\|D \Delta V(t)\|_{2}^{\frac{3}{8}} \\
\lesssim & \eta_{9} \cdot\left(\varepsilon \cdot\left\|\Delta^{2} V(t)\right\|_{2}^{2}+\frac{1}{\varepsilon} \cdot\|D \Delta V(t)\|_{2}^{3}\right)+\eta_{9} \cdot\left(\varepsilon \cdot\left\|\Delta^{2} V(t)\right\|_{2}^{2}+\frac{1}{\varepsilon} \cdot\|D \Delta V(t)\|_{2}^{\frac{9}{4}}\right)
\end{aligned}
$$




$$
\begin{aligned}
& +\eta_{9} \cdot\left(\varepsilon \cdot\left\|\Delta^{2} V(t)\right\|_{2}^{2}+\frac{1}{\varepsilon} \cdot\|D \Delta V(t)\|_{2}^{\frac{3}{4}}\right) \\
\lesssim & \eta_{9} \cdot\left(\varepsilon \cdot\left\|\Delta^{2} V(t)\right\|_{2}^{2}+\frac{1}{\varepsilon} \cdot\|D \Delta V(t)\|_{2}^{4}+\frac{1}{\varepsilon}\right),
\end{aligned}
$$

where $\eta_{9}$ depends on $\mathcal{R}^{M}, \mathcal{R}^{E}$ and their covariant derivatives, $\left\|V_{0}\right\|_{\infty},\left\|V_{0}\right\|_{2},\left\|D V_{0}\right\|_{2}$, $\left\|\Delta V_{0}\right\|_{2}$ and $t$.

Moreover, there is a universal constant $\kappa_{1}$ such that

$$
\begin{aligned}
& \left|\int_{M}\left\langle\Delta\left[|V(t)|^{2} \cdot V(t)\right], \Delta^{2} V(t)\right\rangle d M\right| \\
\leqslant & \frac{1}{4}\left\|\Delta^{2} V(t)\right\|_{2}^{2}+\kappa_{1} \cdot\|V(t)\|_{\infty}^{4} \cdot\|\Delta V(t)\|_{2}^{2}+\kappa_{1} \cdot\|V(t)\|_{\infty}^{2} \cdot\|D V(t)\|_{4}^{4} .
\end{aligned}
$$

Recalling (5.9) and (5.13) we obtain

$$
\begin{aligned}
& \left|\int_{M}\left\langle\Delta\left[|V(t)|^{2} \cdot V(t)\right], \Delta^{2} V(t)\right\rangle d M\right| \leqslant \frac{1}{4}\left\|\Delta^{2} V(t)\right\|_{2}^{2}+\kappa_{1} \cdot\left\|V_{0}\right\|_{\infty}^{4} \cdot \gamma_{5} \\
& +\kappa_{1} \cdot\left\|V_{0}\right\|_{\infty}^{2} \cdot \eta_{4} \cdot\left(\|D \Delta V(t)\|_{2}+\|\Delta V(t)\|_{2}+\|D V(t)\|_{2}+\|V(t)\|_{2}\right) \cdot\|D V(t)\|_{2}^{3} .
\end{aligned}
$$

Substituting the upper bounds of $\|\Delta V(t)\|_{2},\|D V(t)\|_{2}$ and $\|V(t)\|_{2}$ into (5.20) gives

$$
\begin{aligned}
& \left|\int_{M}\left\langle\Delta\left[|V(t)|^{2} \cdot V(t)\right], \Delta^{2} V(t)\right\rangle d M\right| \\
\leqslant & \frac{1}{4}\left\|\Delta^{2} V(t)\right\|_{2}^{2}+\kappa_{2} \cdot\left(\|D \Delta V(t)\|_{2}+1\right) \\
\leqslant & \frac{1}{4}\left\|\Delta^{2} V(t)\right\|_{2}^{2}+\frac{\kappa_{2}}{2} \cdot\left(\|D \Delta V(t)\|_{2}^{2}+3\right),
\end{aligned}
$$

where $\kappa_{2}$ relies on $\mathcal{R}^{M}, \mathcal{R}^{E}$ and their covariant derivatives, $\left\|V_{0}\right\|_{\infty},\left\|V_{0}\right\|_{2},\left\|D V_{0}\right\|_{2},\left\|\Delta V_{0}\right\|_{2}$ and $t$.

In conclusion,

$$
\begin{aligned}
& \frac{1}{2} \frac{d}{d t}\|D \Delta V(t)\|_{2}^{2}+\left\|\Delta^{2} V(t)\right\|_{2}^{2}+\lambda \cdot\|D \Delta V(t)\|_{2}^{2} \\
\leqslant & \eta_{9}^{\prime} \cdot\left(\varepsilon \cdot\left\|\Delta^{2} V(t)\right\|_{2}^{2}+\frac{1}{\varepsilon} \cdot\|D \Delta V(t)\|_{2}^{4}+\frac{1}{\varepsilon}\right)+\frac{1}{4}\left\|\Delta^{2} V(t)\right\|_{2}^{2}+\frac{\kappa_{2}}{2} \cdot\left(\|D \Delta V(t)\|_{2}^{2}+3\right),
\end{aligned}
$$

where $\eta_{9}^{\prime}$ depends on $\mathcal{R}^{M}, \mathcal{R}^{E}$ and their covariant derivatives, $\left\|V_{0}\right\|_{\infty},\left\|V_{0}\right\|_{2},\left\|D V_{0}\right\|_{2}$, $\left\|\Delta V_{0}\right\|_{2}$ and $t$. Let $\varepsilon$ be small enough. From Young's inequality it follows that

$$
\frac{1}{2} \frac{d}{d t}\|D \Delta V(t)\|_{2}^{2}+\frac{1}{2}\left\|\Delta^{2} V(t)\right\|_{2}^{2}+\lambda \cdot\|D \Delta V(t)\|_{2}^{2} \leqslant \eta_{10} \cdot\left(1+\|D \Delta V(t)\|_{2}^{4}\right),
$$

where $\eta_{10}$ is dependent of $\eta_{9}$ and $\kappa_{2}$. Since of (5.14), the generalized Gronwall's inequality implies

$$
\|D \Delta V(t)\|_{2}^{2} \leqslant \gamma_{6}
$$

here $\gamma_{6}$ relies on $\left\|D \Delta V_{0}\right\|_{2}, \eta_{10}, \gamma_{4}, \gamma_{5}$ and $t$. Substituting the upper bounds of $\|\Delta V(t)\|_{2}^{2}$ and $\|D \Delta V(t)\|_{2}^{2}$ into (5.1) and (5.4) yields

which implies

$$
\|D V(t)\|_{H^{2}} \leqslant \gamma_{7}
$$

$$
\|D V(t)\|_{\infty} \lesssim \gamma_{7}
$$


where $\gamma_{7}$ relies on $\mathcal{R}^{M}, \mathcal{R}^{E}$ and their covariant derivatives, $\left\|V_{0}\right\|_{\infty},\left\|V_{0}\right\|_{2},\left\|D V_{0}\right\|_{2},\left\|\Delta V_{0}\right\|_{2}$, $\left\|D \Delta V_{0}\right\|_{2}$ and $t$.

Now we return to prove Theorem 1.3. Reviewing (4.1), we know that the key is to estimate

$$
\sum_{i+j=k-1} a_{i j} \cdot\left\|D^{k+1} V\right\|_{2} \cdot\left\|\left|D^{i+1} V\right| \cdot \mid D^{j+1} V\right\|_{2}
$$

Hölder inequality yields

$$
\left\|\left|D^{i+1} V\right| \cdot \mid D^{j+1} V\right\|_{2} \leqslant\left\|D^{i+1} V\right\|_{\frac{2 k-2}{i}} \cdot\left\|D^{j+1} V\right\|_{\frac{2 k-2}{k-1-i}} .
$$

From Theorem 2.4, it follows that

$$
\left\|D^{i+1} V\right\|_{\frac{2 k-2}{i}} \lesssim\left\|D^{k} V\right\|_{2}^{\frac{i}{k-1}} \cdot\|D V\|_{\infty}^{\frac{k-1-i}{k-1}}
$$

and

$$
\left\|D^{j+1} V\right\|_{\frac{2 k-2}{k-1-i}} \lesssim\left\|D^{k} V\right\|_{2}^{\frac{k-1-i}{k-1}} \cdot\|D V\|_{\infty}^{\frac{i}{k-1}}
$$

So one can get

$$
\left\|\left|D^{i+1} V\right| \cdot\left|D^{j+1} V\right|\right\|_{2} \lesssim\left\|D^{k} V\right\|_{2} \cdot\|D V\|_{\infty} .
$$

Substituting (5.21), (4.3), (4.4) and (4.5) into (4.1) we obtain

$$
\begin{aligned}
& \frac{1}{2} \frac{d}{d t}\left\|D^{k} V\right\|_{2}^{2}+\left\|D^{k+1} V\right\|_{2}^{2} \\
\leqslant & \kappa_{3} \cdot\left\|D^{k+1} V\right\|_{2} \cdot\left\|D^{k} V\right\|_{2} \cdot\|D V\|_{\infty}+G_{k} \cdot\left(1+\left\|V_{0}\right\|_{\infty}+\left\|V_{0}\right\|_{\infty}^{2}\right) \cdot\|V\|_{H^{k}}^{2} \\
\leqslant & \frac{1}{2} \cdot\left\|D^{k+1} V\right\|_{2}^{2}+\frac{\kappa_{3}^{2}}{2} \cdot\left\|D^{k} V\right\|_{2}^{2} \cdot\|D V\|_{\infty}^{2}+G_{k} \cdot\left(1+\left\|V_{0}\right\|_{\infty}+\left\|V_{0}\right\|_{\infty}^{2}\right) \cdot\|V\|_{H^{k}}^{2},
\end{aligned}
$$

where $\kappa_{3}$ is a universal constant. Note the fact

$$
\|D V(t)\|_{\infty} \lesssim \gamma_{7} \quad \forall t \in\left[0, T^{*}\right)
$$

Summing $k$ from 0 to $N$ and applying Gronwall's inequality we are led to

$$
\|V(t)\|_{H^{N}}^{2}+\int_{0}^{t}\|V(s)\|_{H^{N+1}}^{2} d s \leqslant C_{N}\left(\left\|V_{0}\right\|_{H^{N}}, t, \gamma_{7},\left\|V_{0}\right\|_{\infty}, \kappa_{3}, G_{0}, \cdots, G_{N}\right) .
$$

The remaining part of the proof of Theorem 1.3 is as the same as that of Theorem 1.2 . So we omit it. This completes the proof.

\section{REFERENCES}

[1] Charles Baker, The mean curvature flow of submanifolds of high codimension, Ph.D. thesis, Australian National University (2010); arXiv: 1104.4409.

[2] Richard S. Hamilton, Three-manifolds with positive Ricci curvature, J. Differential. Geometry, 17 (1982) 255-306.

[3] Weiyue Ding, Youde Wang; Local Schrödinger flow into Kähler manifolds, Science in China, Series A, 2001, Vol. 44, No. 11.

[4] Boling Guo, Qiaoxin Li, Ming Zeng, Global smooth solutions of the Landau-Lifshitz-Bloch equation, preprint.

[5] Zonglin Jia, Local strong solution to General Landau-Lifshitz-Bloch equation, arXiv:1802.00144. 
[6] Kim Ngan Le, Weak solutions of the Landau-Lifshitz Bloch equation, J. Differential Equations 261 (2016) 6699-6717

\section{Boling Guo}

Institute of Applied Physics and Computational Mathematics, China Academy of Engineering Physics, Beijing, 100088, P. R. China

Email: gbl@iapcm.ac.cn

Zonglin Jia

Institute of Applied Physics and Computational Mathematics, China Academy of Engineering Physics, Beijing, 100088, P. R. China

Email:756693084@qq.com 